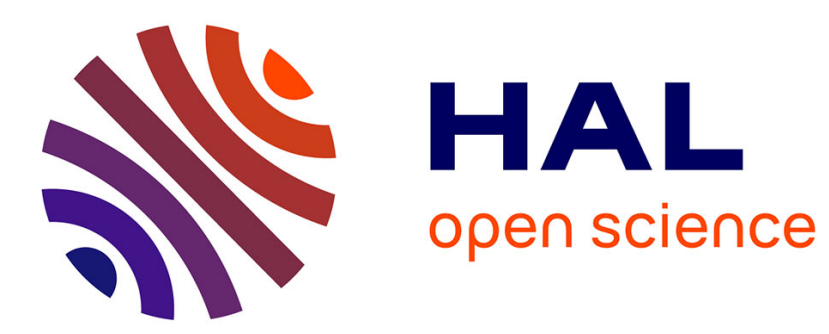

\title{
Robust nonparametric estimation of the conditional tail dependence coefficient
}

\author{
Yuri Goegebeur, Armelle Guillou, Nguyen Khanh Le Ho, Jing Qin
}

\section{To cite this version:}

Yuri Goegebeur, Armelle Guillou, Nguyen Khanh Le Ho, Jing Qin. Robust nonparametric estimation of the conditional tail dependence coefficient. Journal of Multivariate Analysis, 2020, 178, 10.1016/j.jmva.2020.104607 . hal-02269476

\section{HAL Id: hal-02269476 https://hal.science/hal-02269476}

Submitted on 22 Aug 2019

HAL is a multi-disciplinary open access archive for the deposit and dissemination of scientific research documents, whether they are published or not. The documents may come from teaching and research institutions in France or abroad, or from public or private research centers.
L'archive ouverte pluridisciplinaire HAL, est destinée au dépôt et à la diffusion de documents scientifiques de niveau recherche, publiés ou non, émanant des établissements d'enseignement et de recherche français ou étrangers, des laboratoires publics ou privés. 


\title{
Robust nonparametric estimation of the conditional tail dependence coefficient
}

\author{
Yuri Goegebeur $^{(1)}$, Armelle Guillou ${ }^{(2)}$, Nguyen Khanh Le Ho ${ }^{(1)}$, Jing Qin ${ }^{(1)}$ \\ (1) Department of Mathematics and Computer Science, University of Southern Denmark, Campusvej \\ 55, 5230 Odense M, Denmark \\ (2) Institut Recherche Mathématique Avancée, UMR 7501, Université de Strasbourg et CNRS, 7 rue \\ René Descartes, 67084 Strasbourg cedex, France
}

\begin{abstract}
We consider robust and nonparametric estimation of the coefficient of tail dependence in presence of random covariates. The estimator is obtained by fitting the extended Pareto distribution locally to properly transformed bivariate observations using the minimum density power divergence criterion. We establish convergence in probability and asymptotic normality of the proposed estimator under some regularity conditions. The finite sample performance is evaluated with a small simulation experiment, and the practical applicability of the method is illustrated on a real dataset of air pollution measurements.

Keywords: Coefficient of tail dependence, robustness, local estimation, empirical process.
\end{abstract}

\section{Introduction}

Many problems involving extreme events are inherently multivariate, and hence they should be handled with appropriate multivariate extreme value methods. Of particular interest is the estimation of the extremal dependence between two or more variables. A full characterization of the extremal dependence between variables can be obtained from functions like the spectral distribution function or the Pickands dependence function. We refer to Beirlant et al. (2004), and de Haan and Ferreira (2006), and the references therein, for more details about this approach. Alternatively, similar to classical statistics one can try and summarize the extremal dependency in a number of well-chosen coefficients that give a representative picture of the full dependency structure, like, e.g., the coefficient of tail dependence (Ledford and Tawn, 1997). Modelling tail dependence is a critical issue in many scientific disciplines. For instance, in finance and actuarial science an important problem is to estimate very large quantiles of the distribution of the sums of possibly dependent risks (Barbe et al., 2006). In environmental science, studying dependence in extreme levels of pollutants like ozone, particulate matter, carbon monoxide and temperature is important as combined high levels of these variables may pose a major threat to human health (Escobar-Bach et al., 2018). In this paper, we will consider robust and nonparametric estimation of the coefficient of tail dependence when there are random covariates. 
Let $\left(Y^{(1)}, Y^{(2)}\right)$ be a bivariate random vector recorded along with a random covariate $X \in \mathbb{R}^{p}$. The covariate $X$ has density function $f_{X}$ with support $S_{X} \subset \mathbb{R}^{p}$, having non-empty interior. The continuous conditional marginal distribution functions of $Y^{(j)}$ given $X=x$ are denoted by $F_{j}(\cdot \mid x), j=1,2$, and the joint conditional distribution function of the pair satisfies that for all $x \in S_{X}$ and $y \in[0,1]$

$$
\mathbb{P}\left(1-F_{1}\left(Y^{(1)} \mid x\right)<y, 1-F_{2}\left(Y^{(2)} \mid x\right)<y \mid X=x\right)=C(x) y^{\frac{1}{\eta(x)}}\left(1+\frac{1}{\eta(x)} \delta(y \mid x)\right),
$$

where $\eta(x) \in(0,1]$ is the conditional tail dependence coefficient, and $|\delta(\cdot \mid x)|$ is a regularly varying function in the neighborhood of zero with index $\tau(x)>0$. In this paper we focus on the estimation of $\eta(x)$, and introduce a nonparametric estimator, which is obtained from local fits of the above model in a neigborhood of $x$, a point of interest in the covariate space. In absence of covariates, several estimators for $\eta$ have been introduced in the extreme value literature. We refer to Ledford and Tawn (1997), Peng (1999), Draisma et al. (2004), Beirlant et al. (2011), and Goegebeur and Guillou (2013), to name but a few.

Our aim in this paper is to estimate the conditional tail dependence coefficient in a robust way, in order to ensure that our estimation procedure works in the presence of possible outliers. To achieve this, we will use the idea of the density power divergence introduced by Basu et al. (1998). In particular, the density power divergence between density functions $h$ and $g$ is given by

$$
\Delta_{\alpha}(h, g):= \begin{cases}\int_{\mathbb{R}}\left[g^{1+\alpha}(y)-\left(1+\frac{1}{\alpha}\right) g^{\alpha}(y) h(y)+\frac{1}{\alpha} h^{1+\alpha}(y)\right] d y, & \alpha>0 \\ \int_{\mathbb{R}} \log \frac{h(y)}{g(y)} h(y) d y, & \alpha=0 .\end{cases}
$$

Here $h$ is assumed to be the true (typically unknown) density of the data, whereas $g$ is a parametric model, depending on a parameter vector $\theta$ which is determined by minimizing the empirical version of (1). The resulting estimator will be called, in the sequel, minimum density power divergence (MDPD) estimator. In the present paper we will adjust this criterion to the local estimation context with focus on estimating conditional extreme dependence. Dutang et al. (2014) used this criterion to obtain a robust estimator for $\eta$, but in a context without covariates. To the best of our knowledge, robust nonparametric estimation of the conditional coefficient of tail dependence has not been considered so far in the extreme value literature.

The remainder of the paper is organized as follows. In Section 2, we simplify the problem to the case where the conditional marginal distributions are known and we prove the existence, convergence in probability and asymptotic normality of the MDPD estimator of the conditional tail dependence coefficient. Then in Section 3, the realistic situation where the margins are unknown is considered and similar results are established. The efficiency and robustness of our MDPD estimator are illustrated in a small simulation study in Section 4 and on a real dataset on air pollution in Section 5. Finally, all the proofs are postponed to the appendix. 


\section{Case of known margins}

In this section, we assume that the conditional marginal distribution functions $F_{1}(\cdot \mid x)$ and $F_{2}(\cdot \mid x)$ are known.

Define $Z:=\min \left(\frac{1}{1-F_{1}\left(Y^{(1)} \mid X\right)}, \frac{1}{1-F_{2}\left(Y^{(2)} \mid X\right)}\right)$. Direct computations yield for all $x \in S_{X}$

$$
\bar{F}_{Z}(z \mid x):=\mathbb{P}(Z>z \mid X=x)=C(x) z^{-\frac{1}{\eta(x)}}\left(1+\frac{1}{\eta(x)} \delta_{Z}(z \mid x)\right),
$$

where

$$
\delta_{Z}(z \mid x):=\delta\left(\frac{1}{z} \mid x\right) .
$$

Here $\left|\delta_{Z}(\cdot \mid x)\right|$ is a regularly varying function at infinity with index $-\tau(x)$, which is additionally assumed to be normalized, i.e., such that

$$
\delta_{Z}(z \mid x)=A(x) \exp \left(\int_{1}^{z} \frac{\varepsilon(u \mid x)}{u} d u\right)
$$

with $A(x) \in \mathbb{R}$ and $\varepsilon(z \mid x) \rightarrow-\tau(x)$ as $z \rightarrow \infty$.

Note that the conditional distribution of $Z$, given $X=x$, satisfies Condition $(\mathcal{R})$ in Dierckx et al. (2014) with second order parameter $\rho(x):=-\tau(x) \eta(x)$. Thus, one can approximate the conditional distribution of $Z / u$, given $Z>u$, where $u$ denotes a high threshold value, by the extended Pareto distribution given by

$$
G(z ; \eta, \delta, \rho)= \begin{cases}1-\left[z\left(1+\delta-\delta z^{\frac{\rho}{\eta}}\right)\right]^{-\frac{1}{\eta}}, & z>1 \\ 0, & z \leqslant 1\end{cases}
$$

and density function

$$
g(z ; \eta, \delta, \rho)= \begin{cases}\frac{z^{-\frac{1}{\eta}-1}}{\eta}\left[1+\delta\left(1-z^{\frac{\rho}{\eta}}\right)\right]^{-\frac{1}{\eta}-1}\left[1+\delta\left(1-\left(1+\frac{\rho}{\eta}\right) z^{\frac{\rho}{\eta}}\right)\right], & z>1 \\ 0, & z \leqslant 1\end{cases}
$$

where $\eta>0, \rho<0$, and $\delta>\max \{-1, \eta / \rho\}$.

Indeed, as shown in Beirlant et al. (2009), we have

$$
\sup _{z \geqslant 1}\left|\frac{\bar{F}_{Z}(u z \mid x)}{\bar{F}_{Z}(u \mid x)}-\bar{G}\left(z ; \eta(x), \delta_{Z}(u \mid x), \rho(x)\right)\right|=o\left(\delta_{Z}(u \mid x)\right) \text { if } u \rightarrow \infty .
$$

Clearly, based on this result, one can obtain an estimator for $\eta(x)$ by fitting the extended Pareto distribution to the relative excesses over a high threshold. 
Let $\left(X_{1}, Z_{1}\right), \ldots,\left(X_{n}, Z_{n}\right)$ be independent copies of the random vector $(X, Z)$. We develop a nonparametric, robust and asymptotically unbiased estimator for $\eta(x)$ by fitting $g$ locally to the relative excesses $Z_{i} / u_{n}, i=1, \ldots, n$, by means of the MDPD criterion, adjusted to locally weighted estimation, i.e., we minimize

$\widehat{\Delta}_{\alpha}\left(\eta, \delta_{Z} ; \rho\right):=\frac{1}{n} \sum_{i=1}^{n} K_{h_{n}}\left(x-X_{i}\right)\left\{\int_{1}^{\infty} g^{1+\alpha}\left(z ; \eta, \delta_{Z}, \rho\right) d z-\left(1+\frac{1}{\alpha}\right) g^{\alpha}\left(\frac{Z_{i}}{u_{n}} ; \eta, \delta_{Z}, \rho\right)\right\} \mathbb{1}_{\left\{Z_{i}>u_{n}\right\}}$,

in case $\alpha>0$ and

$$
\widehat{\Delta}_{0}\left(\eta, \delta_{Z} ; \rho\right):=-\frac{1}{n} \sum_{i=1}^{n} K_{h_{n}}\left(x-X_{i}\right) \ln g\left(\frac{Z_{i}}{u_{n}} ; \eta, \delta_{Z}, \rho\right) \mathbb{1}_{\left\{Z_{i}>u_{n}\right\}},
$$

in case $\alpha=0$, where $K_{h_{n}}(x):=K\left(x / h_{n}\right) / h_{n}^{p}, K$ is a joint density function on $\mathbb{R}^{p}, h_{n}$ is a nonrandom sequence of bandwidths with $h_{n} \rightarrow 0$ if $n \rightarrow \infty, \mathbb{1}_{\{A\}}$ is the indicator function on the event $A$ and $u_{n}$ is a local non-random threshold sequence satisfying $u_{n} \rightarrow \infty$ if $n \rightarrow \infty$. Note that in case $\alpha=0$, the local empirical density power divergence criterion corresponds with a locally weighted log-likelihood function. The parameter $\alpha$ controls the trade-off between efficiency and robustness of the MDPD criterion: the estimator becomes more efficient but less robust as $\alpha$ gets closer to zero, whereas for increasing $\alpha$ the robustness increases and the efficiency decreases. Note that we only estimate $\eta(x)$ and $\delta_{Z}\left(u_{n} \mid x\right)$ with the MDPD criterion, while the second order parameter $\rho(x)$ will be fixed at some value. Fixing second order parameters like $\rho(x)$ here at some value is a common practice in extreme value statistics, and was also proposed in Beirlant et al. (1999), Feuerverger and Hall (1999), and Gomes and Martins (2004). Alternatively, one can replace $\rho(x)$ by an external consistent estimator. However, the estimation of $\rho(x)$ in a robust way is still an open problem, and moreover, using an external consistent estimator rather than a canonical value, does not, in general, improve the performance of the final MDPD estimator in practice. For all these reasons, we only use a canonical value for the parameter $\rho(x)$ in the sequel.

The MDPD estimators of $\left(\eta(x), \delta_{Z}\left(u_{n} \mid x\right)\right)$ satisfy the estimating equations

$$
\begin{aligned}
0= & \frac{1}{n} \sum_{i=1}^{n} K_{h_{n}}\left(x-X_{i}\right) \mathbb{1}_{\left\{Z_{i}>u_{n}\right\}} \int_{1}^{\infty} g^{\alpha}\left(z ; \eta, \delta_{Z}, \rho\right) \frac{\partial g\left(z ; \eta, \delta_{Z}, \rho\right)}{\partial \eta} d z \\
& -\frac{1}{n} \sum_{i=1}^{n} K_{h_{n}}\left(x-X_{i}\right) g^{\alpha-1}\left(\frac{Z_{i}}{u_{n}} ; \eta, \delta_{Z}, \rho\right) \frac{\partial g\left(\frac{Z_{i}}{u_{n}} ; \eta, \delta_{Z}, \rho\right)}{\partial \eta} \mathbb{1}_{\left\{Z_{i}>u_{n}\right\}}, \\
0= & \frac{1}{n} \sum_{i=1}^{n} K_{h_{n}}\left(x-X_{i}\right) \mathbb{1}_{\left\{Z_{i}>u_{n}\right\}} \int_{1}^{\infty} g^{\alpha}\left(z ; \eta, \delta_{Z}, \rho\right) \frac{\partial g\left(z ; \eta, \delta_{Z}, \rho\right)}{\partial \delta_{Z}} d z \\
& -\frac{1}{n} \sum_{i=1}^{n} K_{h_{n}}\left(x-X_{i}\right) g^{\alpha-1}\left(\frac{Z_{i}}{u_{n}} ; \eta, \delta_{Z}, \rho\right) \frac{\partial g\left(\frac{Z_{i}}{u_{n}} ; \eta, \delta_{Z}, \rho\right)}{\partial \delta_{Z}} \mathbb{1}_{\left\{Z_{i}>u_{n}\right\}} .
\end{aligned}
$$

The following statistic is crucial for studying the asymptotic behavior of the estimators. Set $\ln _{+} x:=\ln \max \{x, 1\}, x>0$, and

$$
T_{n}(K, s, t \mid x):=\frac{1}{n} \sum_{i=1}^{n} K_{h_{n}}\left(x-X_{i}\right)\left(\frac{Z_{i}}{u_{n}}\right)^{s}\left(\ln _{+} \frac{Z_{i}}{u_{n}}\right)^{t} 1_{\left\{Z_{i}>u_{n}\right\}}
$$


where $s \leqslant 0$ and $t \geqslant 0$. The motivation for considering this type of statistic is that the estimating equations (4) and (5) only depend on statistics of this form. Note that $\ln _{+} x$ is introduced to ensure that $\left(\ln _{+} Z_{i} / u_{n}\right)^{t}$ is always well defined ( $t$ is nonnegative, not necessary integer).

Due to the regression context, we need the following classical Hölder-type conditions. Here $\|\cdot\|$ denotes some norm on $\mathbb{R}^{p}$.

Assumption $(\mathcal{H})$ There exist positive constants $M_{f_{X}}, M_{C}, M_{A}, M_{\eta}, M_{\varepsilon}, \delta_{f_{X}}, \delta_{C}, \delta_{A}, \delta_{\eta}$, and $\delta_{\varepsilon}$, such that for all $(x, z) \in S_{X} \times S_{X}$ :

$$
\begin{aligned}
\left|f_{X}(x)-f_{X}(z)\right| & \leqslant M_{f_{X}}\|x-z\|^{\delta_{f_{X}}}, \\
|C(x)-C(z)| & \leqslant M_{C}\|x-z\|^{\delta_{C}}, \\
|A(x)-A(z)| & \leqslant M_{A}\|x-z\|^{\delta_{A}}, \\
|\eta(x)-\eta(z)| & \leqslant M_{\eta}\|x-z\|^{\delta_{\eta}}, \\
\sup _{y \geqslant 1}|\varepsilon(y \mid x)-\varepsilon(y \mid z)| & \leqslant M_{\varepsilon}\|x-z\|^{\delta_{\varepsilon}} .
\end{aligned}
$$

Also, the following assumption, standard in the context of local estimation, is required on the kernel function.

Assumption $\left(\mathcal{K}_{1}\right) K$ is a bounded density function on $\mathbb{R}^{p}$, with support $S_{K}$ included in the unit ball of $\mathbb{R}^{p}$.

In order to establish the asymptotic normality of the consistent sequence of solutions $\left(\widehat{\eta}_{n}(x), \widehat{\delta}_{Z, n}(x)\right)$ of the estimating equations (4) and (5), we introduce

$$
\mathbb{S}_{n}^{(j)}(s):=\sqrt{n h_{n}^{p} \bar{F}_{Z}\left(u_{n} \mid x\right) f_{X}(x)}\left[\frac{T_{n}(K, s, j \mid x)}{\bar{F}_{Z}\left(u_{n} \mid x\right) f_{X}(x)}-\frac{j ! \eta_{0}^{j}(x)}{\left[1-s \eta_{0}(x)\right]^{j+1}}\right], j \in\{0,1,2,3\},
$$

where we denote by $\eta_{0}(x)$, resp. $\rho_{0}(x)$, the true conditional tail dependence coefficient, resp. second order parameter.

Theorem 2.1 Let $\left(X_{1}, Z_{1}\right), \ldots,\left(X_{n}, Z_{n}\right)$ be a sample of independent copies of the random vector $(X, Z)$ where the distribution of $Z$, given $X=x$, satisfies (2) and (3), $X$ follows a distribution with density function $f_{X}$, and assume $(\mathcal{H})$ and $\left(\mathcal{K}_{1}\right)$ hold. For all $x \in \operatorname{Int}\left(S_{X}\right)$ with $f_{X}(x)>$ 0 , we assume that $u_{n} \rightarrow \infty$ and $h_{n} \rightarrow 0$ in such a way that $h_{n}^{\delta_{\varepsilon}} \ln u_{n} \rightarrow 0, n h_{n}^{p} \bar{F}_{Z}\left(u_{n} \mid x\right) \rightarrow \infty$, $\sqrt{n h_{n}^{p} \bar{F}_{Z}\left(u_{n} \mid x\right)} \delta_{Z}\left(u_{n} \mid x\right) \rightarrow \lambda \in \mathbb{R}, \sqrt{n h_{n}^{p} \bar{F}_{Z}\left(u_{n} \mid x\right)} h_{n}^{\delta_{f_{X}} \wedge \delta_{C}} \rightarrow 0, \sqrt{n h_{n}^{p} \bar{F}_{Z}\left(u_{n} \mid x\right)} h_{n}^{\delta_{\eta}} \ln u_{n} \rightarrow 0$. Then in $\mathcal{C}^{4}([S, 0]), S<0$,

$$
\left(\mathbb{S}_{n}^{(0)}, \mathbb{S}_{n}^{(1)}, \mathbb{S}_{n}^{(2)}, \mathbb{S}_{n}^{(3)}\right) \rightsquigarrow\left(\mathbb{S}^{(0)}, \mathbb{S}^{(1)}, \mathbb{S}^{(2)}, \mathbb{S}^{(3)}\right), \quad \text { for } n \rightarrow \infty,
$$

a Gaussian process, with, for $s \in[S, 0]$, mean functions

$$
\mathbb{E}\left[\mathbb{S}^{(j)}(s)\right]=-\lambda \sqrt{f_{X}(x)} j ! \eta_{0}^{j-1}(x)\left[\frac{1}{\left[1-s \eta_{0}(x)\right]^{j+1}}-\frac{1-\rho_{0}(x)}{\left[1-\rho_{0}(x)-s \eta_{0}(x)\right]^{j+1}}\right], \quad j \in\{0,1,2,3\},
$$


and covariance functions given by

$$
\operatorname{Cov}\left(\mathbb{S}^{(j)}\left(s_{1}\right), \mathbb{S}^{(k)}\left(s_{2}\right)\right)=\frac{(j+k) ! \eta_{0}^{j+k}(x)\|K\|_{2}^{2}}{\left[1-\left(s_{1}+s_{2}\right) \eta_{0}(x)\right]^{1+j+k}}, \quad(j, k) \in\{0,1,2,3\}^{2} .
$$

Note that Dierckx et al. (2014) obtained a similar result, though under their high level assumption called $(\mathcal{M})$, which is avoided in the present paper.

Based on this theorem, one can now establish the existence, convergence in probability and asymptotic normality of the MDPD estimators of $\left(\eta_{0}(x), \delta_{Z}\left(u_{n} \mid x\right)\right)$, when suitably normalized. This theorem is similar to Theorems 2 and 3 in Dierckx et al. (2014) with our new conditions given in our Theorem 2.1, and thus the proof is omitted.

Theorem 2.2 Let $\left(X_{1}, Z_{1}\right), \ldots,\left(X_{n}, Z_{n}\right)$ be a sample of independent copies of the random vector $(X, Z)$ where the distribution of $Z$, given $X=x$, satisfies (2) and (3), $X$ follows a distribution with density function $f_{X}$, and assume $(\mathcal{H})$ and $\left(\mathcal{K}_{1}\right)$ hold.

For all $x \in \operatorname{Int}\left(S_{X}\right)$ with $f_{X}(x)>0$, let $u_{n} \rightarrow \infty$ and $h_{n} \rightarrow 0$ in such a way that $n h_{n}^{p} \bar{F}_{Z}\left(u_{n} \mid x\right) \rightarrow$ $\infty$ and $h_{n}^{\delta_{\eta} \wedge \delta_{\varepsilon}} \ln u_{n} \rightarrow 0$. Then with probability tending to 1 , there exists sequences of solutions $\left(\widehat{\eta}_{n}(x), \widehat{\delta}_{Z, n}(x)\right)$ of the estimating equations (4) and (5), with $\rho$ fixed at $\rho_{0}(x)$ such that $\left(\widehat{\eta}_{n}(x), \widehat{\delta}_{Z, n}(x)\right) \stackrel{\mathbb{P}}{\longrightarrow}\left(\eta_{0}(x), 0\right)$.

If additionally,

$$
\begin{aligned}
& \sqrt{n h_{n}^{p} \bar{F}_{Z}\left(u_{n} \mid x\right)} \delta_{Z}\left(u_{n} \mid x\right) \longrightarrow \lambda \in \mathbb{R}, \\
& \sqrt{n h_{n}^{p} \bar{F}_{Z}\left(u_{n} \mid x\right)} h_{n}^{\delta_{f_{X}} \wedge \delta_{C}} \longrightarrow 0, \\
& \sqrt{n h_{n}^{p} \bar{F}_{Z}\left(u_{n} \mid x\right)} h_{n}^{\delta_{\eta}} \ln u_{n} \longrightarrow 0,
\end{aligned}
$$

then

$\sqrt{n h_{n}^{p} \bar{F}_{Z}\left(u_{n} \mid x\right) f_{X}(x)}\left[\begin{array}{c}\hat{\eta}_{n}(x)-\eta_{0}(x) \\ \hat{\delta}_{Z, n}(x)-\delta_{Z}\left(u_{n} \mid x\right)\end{array}\right] \rightsquigarrow \quad N_{2}\left(\mathbf{0}, \mathbb{C}^{-1}\left(\rho_{0}(x)\right) \mathbb{B}\left(\rho_{0}(x)\right) \boldsymbol{\Sigma}\left(\rho_{0}(x)\right) \mathbb{B}^{\prime}\left(\rho_{0}(x)\right) \mathbb{C}^{-1}\left(\rho_{0}(x)\right)\right)$,

for $n \rightarrow \infty$, where the matrix $\mathbb{B}\left(\rho_{0}(x)\right)$ is defined by

$$
\mathbb{B}\left(\rho_{0}(x)\right):=\eta_{0}^{-\alpha-2}(x)\left[\begin{array}{cccc}
-\frac{\alpha \eta_{0}(x)\left(1+\eta_{0}(x)\right)}{\left[1+\alpha\left(1+\eta_{0}(x)\right)\right]^{2}} & \eta_{0}(x) & 0 & -1 \\
-\frac{\alpha \eta_{0}(x) \rho_{0}(x)\left(1+\eta_{0}(x)\right)}{\left[1+\alpha\left(1+\eta_{0}(x)\right)\right]\left[1-\rho_{0}(x)+\alpha\left(1+\eta_{0}(x)\right)\right]} & \eta_{0}(x) & -\eta_{0}(x)\left(1-\rho_{0}(x)\right) & 0
\end{array}\right],
$$


the elements of the symmetric $(4 \times 4)$ matrix $\boldsymbol{\Sigma}\left(\rho_{0}(x)\right)$ are given by

$$
\begin{aligned}
\sigma_{11}\left(\rho_{0}(x)\right) & :=\|K\|_{2}^{2} \\
\sigma_{21}\left(\rho_{0}(x)\right) & :=\frac{\|K\|_{2}^{2}}{1+\alpha\left(1+\eta_{0}(x)\right)} \\
\sigma_{22}\left(\rho_{0}(x)\right) & :=\frac{\|K\|_{2}^{2}}{1+2 \alpha\left(1+\eta_{0}(x)\right)} \\
\sigma_{31}\left(\rho_{0}(x)\right) & :=\frac{\|K\|_{2}^{2}}{1-\rho_{0}(x)+\alpha\left(1+\eta_{0}(x)\right)} \\
\sigma_{32}\left(\rho_{0}(x)\right) & :=\frac{\|K\|_{2}^{2}}{1-\rho_{0}(x)+2 \alpha\left(1+\eta_{0}(x)\right)} \\
\sigma_{33}\left(\rho_{0}(x)\right) & :=\frac{\|K\|_{2}^{2}}{1-2 \rho_{0}(x)+2 \alpha\left(1+\eta_{0}(x)\right)} \\
\sigma_{41}\left(\rho_{0}(x)\right) & :=\frac{\eta_{0}(x)\|K\|_{2}^{2}}{\left[1+\alpha\left(1+\eta_{0}(x)\right)\right]^{2}} \\
\sigma_{42}\left(\rho_{0}(x)\right) & :=\frac{\eta_{0}(x)\|K\|_{2}^{2}}{\left[1+2 \alpha\left(1+\eta_{0}(x)\right)\right]^{2}} \\
\sigma_{43}\left(\rho_{0}(x)\right) & :=\frac{\eta_{0}(x)\|K\|_{2}^{2}}{\left[1-\rho_{0}(x)+2 \alpha\left(1+\eta_{0}(x)\right)\right]^{2}} \\
\sigma_{44}\left(\rho_{0}(x)\right) & :=\frac{2 \eta_{0}^{2}(x)\|K\|_{2}^{2}}{\left[1+2 \alpha\left(1+\eta_{0}(x)\right)\right]^{3}}
\end{aligned}
$$

and those of the symmetric $(2 \times 2)$ matrix $\mathbb{C}\left(\rho_{0}(x)\right)$ by

$$
\begin{aligned}
C_{11}\left(\rho_{0}(x)\right) & :=\eta_{0}^{-\alpha-2}(x) \frac{1+\alpha^{2}\left(1+\eta_{0}(x)\right)^{2}}{\left[1+\alpha\left(1+\eta_{0}(x)\right)\right]^{3}} \\
C_{21}\left(\rho_{0}(x)\right) & :=\eta_{0}^{-\alpha-2}(x) \frac{\rho_{0}(x)\left(1-\rho_{0}(x)\right)\left[1+\alpha\left(1+\eta_{0}(x)\right)+\alpha^{2}\left(1+\eta_{0}(x)\right)^{2}\right]+\alpha^{3} \rho_{0}(x)\left(1+\eta_{0}(x)\right)^{3}}{\left[1+\alpha\left(1+\eta_{0}(x)\right)\right]^{2}\left[1-\rho_{0}(x)+\alpha\left(1+\eta_{0}(x)\right)\right]^{2}} \\
C_{22}\left(\rho_{0}(x)\right) & :=\eta_{0}^{-\alpha-2}(x) \frac{\left(1-\rho_{0}(x)\right) \rho_{0}^{2}(x)+\alpha \rho_{0}^{2}(x)\left(1+\eta_{0}(x)\right)\left[\alpha\left(1+\eta_{0}(x)\right)-\rho_{0}(x)\right]}{\left[1+\alpha\left(1+\eta_{0}(x)\right)\right]\left[1-\rho_{0}(x)+\alpha\left(1+\eta_{0}(x)\right)\right]\left[1-2 \rho_{0}(x)+\alpha\left(1+\eta_{0}(x)\right)\right]} .
\end{aligned}
$$

Note that the expected value of the limiting random vector in Theorem 2.2 is zero, whatever the value of $\lambda$. The estimator is therefore said to be asymptotically unbiased.

The following proposition deals with the behavior of the MDPD estimators $\left(\widehat{\eta}_{n}(x), \widehat{\delta}_{Z, n}(x)\right)$ when the parameter $\rho(x)$ is fixed at some value $\widetilde{\rho}<0$, possibly mis-specified.

Proposition 2.1 Under the assumptions of Theorem 2.2, but now with $\rho$ fixed at $\widetilde{\rho}$ in the estimating equation (4) and (5), with probability tending to 1, there exists sequences of solutions $\left(\widehat{\eta}_{n}(x), \widehat{\delta}_{Z, n}(x)\right)$ of the estimating equations such that $\left(\widehat{\eta}_{n}(x), \widehat{\delta}_{Z, n}(x)\right) \stackrel{\mathbb{P}}{\longrightarrow}\left(\eta_{0}(x), 0\right)$. 
If additionally (6), (7) and (8) hold, then

$$
\sqrt{n h_{n}^{p} \bar{F}_{Z}\left(u_{n} \mid x\right) f_{X}(x)}\left[\begin{array}{c}
\hat{\eta}_{n}(x)-\eta_{0}(x) \\
\widehat{\delta}_{Z, n}(x)
\end{array}\right] \rightsquigarrow N_{2}\left(-\lambda \sqrt{f_{X}(x)} \mathbb{C}^{-1}(\widetilde{\rho}) \mathbb{B}(\widetilde{\rho}) \mathbb{D}, \mathbb{C}^{-1}(\widetilde{\rho}) \mathbb{B}(\widetilde{\rho}) \boldsymbol{\Sigma}(\widetilde{\rho}) \mathbb{B}^{\prime}(\widetilde{\rho}) \mathbb{C}^{-1}(\widetilde{\rho})\right),
$$

for $n \rightarrow \infty$, where the elements of the vector $\mathbb{D}$ are the following

$$
\begin{aligned}
& D_{1}:=0 \\
& D_{2}:=-\frac{\alpha \rho_{0}(x)\left(1+\eta_{0}(x)\right)}{\eta_{0}(x)\left[1+\alpha\left(1+\eta_{0}(x)\right)\right]\left[1-\rho_{0}(x)+\alpha\left(1+\eta_{0}(x)\right)\right]} \\
& D_{3}:=-\frac{\left[\alpha\left(1+\eta_{0}(x)\right)-\tilde{\rho}\right] \rho_{0}(x)}{\eta_{0}(x)\left[1-\widetilde{\rho}+\alpha\left(1+\eta_{0}(x)\right)\right]\left[1-\rho_{0}(x)-\widetilde{\rho}+\alpha\left(1+\eta_{0}(x)\right)\right]} \\
& D_{4}:=\frac{\rho_{0}(x)\left(1-\rho_{0}(x)\right)-\alpha^{2} \rho_{0}(x)\left(1+\eta_{0}(x)\right)^{2}}{\left[1+\alpha\left(1+\eta_{0}(x)\right)\right]^{2}\left[1-\rho_{0}(x)+\alpha\left(1+\eta_{0}(x)\right)\right]^{2}} .
\end{aligned}
$$

Again the proof of Proposition 2.1 is similar to the one of Proposition 1 in Dierckx et al. (2014) and thus is omitted. Note that in case $\widetilde{\rho}$ is mis-specified, then the mean of the limiting normal distribution is not necessarily zero, and hence one possible loses the asymptotic unbiasedness. However, as will be clear from the simulations, even though $\widetilde{\rho}$ is mis-specified, the proposed MDPD estimator performs well with respect to bias. Also note that the asymptotic variance expression in Proposition 2.1 is the same as that in Theorem 2.2, though with $\rho_{0}(x)$ replaced by $\widetilde{\rho}$.

\section{Case of unknown margins}

In this section, we consider the general framework where both $F_{1}(\cdot \mid x)$ and $F_{2}(\cdot \mid x)$ are unknown conditional distribution functions. We want to mimic what has been done in the previous section. To this aim, we define

$$
\check{Z}:=\min \left(\frac{1}{1-F_{n, 1}\left(Y^{(1)} \mid X\right)}, \frac{1}{1-F_{n, 2}\left(Y^{(2)} \mid X\right)}\right),
$$

for suitable estimators $F_{n, j}$ of $F_{j}, j=1,2$. Then similarly as in the previous section, the statistic

$$
\check{T}_{n}(K, s, t \mid x):=\frac{1}{n} \sum_{i=1}^{n} K_{h_{n}}\left(x-X_{i}\right)\left(\frac{\check{Z}_{i}}{u_{n}}\right)^{s}\left(\ln _{+} \frac{\check{Z}_{i}}{u_{n}}\right)^{t} 1_{\left\{\check{Z}_{i}>u_{n}\right\}},
$$

is the cornerstone for the MDPD estimator, denoted $\breve{\eta}_{n}(x)$. In particular, the main result relies essentially on the asymptotic properties of this statistic, and so the idea will be to decompose

$$
\sqrt{n h_{n}^{p} \bar{F}_{Z}\left(u_{n} \mid x\right) f_{X}(x)}\left[\frac{\check{T}_{n}(K, s, j \mid x)}{\bar{F}_{Z}\left(u_{n} \mid x\right) f_{X}(x)}-\frac{j ! \eta_{0}^{j}(x)}{\left[1-s \eta_{0}(x)\right]^{j+1}}\right]
$$


into the two terms

$$
\begin{aligned}
& \sqrt{n h_{n}^{p} \bar{F}_{Z}\left(u_{n} \mid x\right) f_{X}(x)}\left[\frac{T_{n}(K, s, j \mid x)}{\bar{F}_{Z}\left(u_{n} \mid x\right) f_{X}(x)}-\frac{j ! \eta_{0}^{j}(x)}{\left[1-s \eta_{0}(x)\right]^{j+1}}\right] \\
& +\sqrt{n h_{n}^{p} \bar{F}_{Z}\left(u_{n} \mid x\right) f_{X}(x)}\left[\frac{\breve{T}_{n}(K, s, j \mid x)}{\bar{F}_{Z}\left(u_{n} \mid x\right) f_{X}(x)}-\frac{T_{n}(K, s, j \mid x)}{\bar{F}_{Z}\left(u_{n} \mid x\right) f_{X}(x)}\right] .
\end{aligned}
$$

The first term can be dealt with using the results from the previous section, whereas we have to show that the second term is negligible for all $s<0$ with $j \in\{0,1,2,3\}$ or $(s, j)=(0,0)$.

In the sequel, we will use the empirical kernel estimator of the unknown distribution functions

$$
F_{n, j}(y \mid x):=\frac{\sum_{i=1}^{n} K_{c}\left(x-X_{i}\right) 1_{\left\{Y_{i}^{(j)} \leqslant y\right\}}}{\sum_{i=1}^{n} K_{c}\left(x-X_{i}\right)}, j=1,2,
$$

where the bandwidth $c:=c_{n}$ is a positive non-random sequence satisfying $c_{n} \rightarrow 0$ as $n \rightarrow \infty$. Here we kept the same kernel $K$ as in the divergence objective function, but of course any other kernel function can be used.

Before stating our main results, we need to impose again some assumptions, in particular a Hölder-type condition on each conditional marginal distribution function $F_{j}$ similar to those imposed in Section 2.

Assumption $(\mathcal{F})$. There exist $M_{F_{j}}>0$ and $\delta_{F_{j}}>0$ such that $\left|F_{j}(y \mid x)-F_{j}(y \mid z)\right| \leqslant M_{F_{j}} \| x-$ $z \|^{\delta_{F_{j}}}$, for all $y \in \mathbb{R}$ and all $(x, z) \in S_{X} \times S_{X}$, and $j=1,2$.

Concerning the kernel $K$ a stronger assumption than $\left(\mathcal{K}_{1}\right)$ is needed. Denote by $B_{z}(r)$ the closed ball with center $z$ and radius $r$ with respect to $\|\cdot\|$.

Assumption $\left(\mathcal{K}_{2}\right) . K$ satisfies Assumption $\left(\mathcal{K}_{1}\right)$, there exists $\delta, m>0$ such that $B_{0}(\delta) \subset S_{K}$ and $K(u) \geqslant m$ for all $u \in B_{0}(\delta)$, and $K$ belongs to the linear span (the set of finite linear combinations) of functions $k \geqslant 0$ satisfying the following property: the subgraph of $k$, $\{(s, u): k(s) \geqslant u\}$, can be represented as a finite number of Boolean operations among sets of the form $\{(s, u): q(s, u) \geqslant \varphi(u)\}$, where $q$ is a polynomial on $\mathbb{R}^{p} \times \mathbb{R}$ and $\varphi$ is an arbitrary real function.

The latter assumption has already been used in Giné and Guillou (2002) or Giné et al. (2004). In particular, it allows us to measure the discrepancy between the conditional distribution function $F_{j}$ and its empirical kernel version $F_{n, j}$, as stated in the following lemma established by EscobarBach et al. (2018).

Lemma 3.1 Assume that there exists $b>0$ such that $f(x) \geqslant b, \forall x \in S_{X} \subset \mathbb{R}^{p}, f$ is bounded, and $\left(\mathcal{K}_{2}\right)$ and $(\mathcal{F})$ hold. Consider a sequence $c$ tending to 0 as $n \rightarrow \infty$ such that for some $q>1$

$$
\frac{|\log c|^{q}}{n c^{p}} \longrightarrow 0
$$


Also assume that there exists an $\varepsilon>0$ such that for $n$ sufficiently large

$$
\inf _{x \in S_{X}} \lambda\left(\left\{u \in B_{0}(1): x-c u \in S_{X}\right\}\right)>\varepsilon,
$$

where $\lambda$ denotes the Lebesgue measure. Then, for any $0<\delta<\min \left(\delta_{F_{1}}, \delta_{F_{2}}\right)$, we have

$$
\sup _{(y, x) \in \mathbb{R} \times S_{X}}\left|F_{n, j}(y \mid x)-F_{j}(y \mid x)\right|=o_{\mathbb{P}}\left(\max \left(\sqrt{\frac{\mid \log c^{q}}{n c^{p}}}, c^{\delta}\right)\right), \text { for } j=1,2 .
$$

We are now able to study the second term in (9).

Theorem 3.1 Let $\left(X_{1}, Z_{1}\right), \ldots,\left(X_{n}, Z_{n}\right)$ be a sample of independent copies of the random vector $(X, Z)$ where the distribution of $Z$, given $X=x$, satisfies (2) and (3), $X$ follows a distribution with a bounded density function $f_{X}$, and such that there exists $b>0$ satisfying $f(x) \geqslant b, \forall x \in S_{X} \subset \mathbb{R}^{p}$. Assume also Assumptions $(\mathcal{H}),\left(\mathcal{K}_{2}\right)$ and $(\mathcal{F})$.

Consider now a sequence $c$ tending to 0 as $n \rightarrow \infty$ such that for some $q>1$

$$
\frac{|\log c|^{q}}{n c^{p}} \longrightarrow 0
$$

Also assume that there exists an $\varepsilon>0$ such that for $n$ sufficiently large

$$
\inf _{x \in S_{X}} \lambda\left(\left\{u \in B_{0}(1): x-c u \in S_{X}\right\}\right)>\varepsilon,
$$

where $\lambda$ denotes the Lebesgue measure. Let $u_{n} \rightarrow \infty$ and $h_{n} \rightarrow 0$ in such a way that for any $\delta \in\left(0, \min \left(\delta_{F_{1}}, \delta_{F_{2}}\right)\right)$

$$
\begin{aligned}
n h_{n}^{p} r_{n}:=n h_{n}^{p} \max \left(\sqrt{\frac{\mid \log c^{q}}{n c^{p}}}, c^{\delta}\right) & \longrightarrow 0 \\
n h_{n}^{p} \bar{F}_{Z}\left(u_{n} \mid x\right) & \longrightarrow \infty,
\end{aligned}
$$

then for any $s<0$ with $j \in\{0,1,2,3\}$ or $(s, j)=(0,0)$, we have

$$
\sqrt{\frac{n h_{n}^{p}}{\bar{F}_{Z}\left(u_{n} \mid x\right) f_{X}(x)}}\left[\breve{T}_{n}(K, s, j \mid x)-T_{n}(K, s, j \mid x)\right]=o_{\mathbb{P}}(1) .
$$

Using Theorem 3.1 we can now establish the main theorem of this paper, stating consistency and asymptotic normality of the conditional $\eta$ estimator, in case of general conditional marginal distribution functions, which are estimated with kernel estimators.

Theorem 3.2 Under the same assumptions as in Theorem 3.1, let $x \in \operatorname{Int}\left(S_{X}\right)$ and suppose that $h_{n}^{\delta_{\eta} \wedge \delta_{\varepsilon}} \ln u_{n} \rightarrow 0$. Then with probability tending to 1 , there exists sequences of solutions $\left(\breve{\eta}_{n}(x), \breve{\delta}_{Z, n}(x)\right)$ of the estimating equations (4) and (5) such that $\left(\breve{\eta}_{n}(x), \check{\delta}_{Z, n}(x)\right) \stackrel{\mathbb{P}}{\longrightarrow}\left(\eta_{0}(x), 0\right)$. If additionally (6), (7) and (8) hold, then

$$
\sqrt{n h_{n}^{p} \bar{F}_{Z}\left(u_{n} \mid x\right) f_{X}(x)}\left[\begin{array}{c}
\check{\eta}_{n}(x)-\eta_{0}(x) \\
\check{\delta}_{Z, n}(x)
\end{array}\right] \rightsquigarrow N_{2}\left(-\lambda \sqrt{f_{X}(x)} \mathbb{C}^{-1}(\widetilde{\rho}) \mathbb{B}(\widetilde{\rho}) \mathbb{D}, \mathbb{C}^{-1}(\widetilde{\rho}) \mathbb{B}(\widetilde{\rho}) \boldsymbol{\Sigma}(\widetilde{\rho}) \mathbb{B}^{\prime}(\widetilde{\rho}) \mathbb{C}^{-1}(\widetilde{\rho})\right)
$$

The result of Theorem 3.2 follows directly from the decomposition (9) and Theorem 3.1, and therefore we omit the proof of it from the paper. 


\section{A simulation study}

Our aim in this section is to illustrate the performance of our robust conditional tail dependence coefficient estimator with a small simulation study in case $p=1$. The joint conditional distribution function of the pair has the following form:

$$
\mathbb{P}\left(1-F_{1}\left(Y^{(1)} \mid x\right)<y_{1}, 1-F_{2}\left(Y^{(2)} \mid x\right)<y_{2} \mid X=x\right)=C\left(y_{1}, y_{2} \mid x\right),
$$

where $C(., . \mid x)$ is one of the three copulas:

Case 1: The BB6 copula in Joe (1997, p. 152) defined for $\theta(x) \geqslant 1$ and $\zeta(x) \geqslant 1$, as follows

$C\left(y_{1}, y_{2} \mid x\right)=1-\left[1-\exp \left\{-\left(\left[-\log \left\{1-\left(1-y_{1}\right)^{\theta(x)}\right\}\right]^{\zeta(x)}+\left[-\log \left\{1-\left(1-y_{2}\right)^{\theta(x)}\right\}\right]^{\zeta(x)}\right)^{\frac{1}{\zeta(x)}}\right\}\right]^{\frac{1}{\theta(x)}}$.

For this model exact independence is obtained for $\theta(x)=1$ with $\zeta(x)=1$, and perfect dependence is achieved if either $\theta(x) \rightarrow \infty$ or $\zeta(x) \rightarrow \infty$. We can easily see that in case $\theta(x)>1$, this model satisfies our model assumption $(2)$ with $\eta(x)=2^{-\frac{1}{\zeta(x)}}, C(x)=[\theta(x)]^{\frac{1}{\zeta(x)}}-1$ and $\tau(x)=1$. We take $X \sim U(1,6), \theta(x)=2$ and $\zeta(x)=x$.

Case 2: The Farlie Gumbel Morgenstern copula defined for $\zeta(x) \in(-1,1]$, as follows

$$
C\left(y_{1}, y_{2} \mid x\right)=y_{1} y_{2}\left[1+\zeta(x)\left(1-y_{1}\right)\left(1-y_{2}\right)\right] .
$$

Exact independence is obtained for $\zeta(x)=0$, and perfect dependence is not attainable under this model. Clearly, for $\zeta(x) \neq 0$, our model assumption (2) is also satisfied, with $\eta(x)=1 / 2$, $C(x)=1+\zeta(x)$ and $\tau(x)=1$. We take $X \sim U(-0.9,1)$ and $\zeta(x)=x$.

Case 3: The BB9 or Crowder copula in Joe (1997, p. 154) defined for $\alpha(x) \geqslant 0$ and $\theta(x) \geqslant 1$, as follows

$$
C\left(y_{1}, y_{2} \mid x\right)=\exp \left(-\left[\left\{\alpha(x)-\log \left(y_{1}\right)\right\}^{\theta(x)}+\left\{\alpha(x)-\log \left(y_{2}\right)\right\}^{\theta(x)}-\alpha^{\theta(x)}\right]^{\frac{1}{\theta(x)}}+\alpha(x)\right) .
$$

Exact independence is obtained for $\theta(x)=1$ or $\alpha(x) \rightarrow \infty$, and perfect dependence for $\theta(x) \rightarrow \infty$. We can check that this model has the form of $(2)$ with $\eta(x)=2^{-\frac{1}{\theta(x)}}, C(x)=$ $\exp \left\{\alpha(x)\left[1-2^{\frac{1}{\theta(x)}}\right]\right\}$, but $\tau(x)=0$. That means that this case does not fit our model assumption, but we use it here to show the robustness of our approach in case our main assumption is violated. We set $X \sim U(1,6), \alpha(x)=1$ and $\theta(x)=x$.

These copula models are combined with unit Fréchet marginal distributions, leading to

$$
F\left(y_{1}, y_{2} \mid x\right)=\exp \left(-1 / y_{1}\right)+\exp \left(-1 / y_{2}\right)-1+C\left(1-\exp \left(-1 / y_{1}\right), 1-\exp \left(-1 / y_{2}\right) \mid x\right) .
$$

Contamination will be introduced according to the following mixture model

$$
F_{\varepsilon}\left(y_{1}, y_{2} \mid x\right)=(1-\varepsilon) F\left(y_{1}, y_{2} \mid x\right)+\varepsilon F_{c}\left(y_{1}, y_{2} \mid x\right),
$$


where $\varepsilon$ denotes the fraction of contamination, and $F_{c}$ is the contaminating distribution function. We take here

$$
F_{c}\left(y_{1}, y_{2} \mid x\right)=e^{-\left(\min \left\{y_{1}, y_{2}\right\}-a\right)^{-1}}, y_{1}, y_{2}>a,
$$

i.e., the distribution function of completely dependent unit Fréchet random variables, translated by $a$. We take for $a$ quantile 0.999 of the unit Fréchet distribution, and consider $\varepsilon=0,5 \%$ and $10 \%$.

Concerning the kernel function $K$, we take the bi-quadratic function

$$
K(x)=\frac{15}{16}\left(1-x^{2}\right)^{2} \mathbb{1}_{\{x \in[-1,1]\}} .
$$

To compute our estimator $\breve{\eta}_{n}(x)$, two sequences $h_{n}$ and $c$ have to be chosen. Concerning $c$, we can use the following cross validation criterion introduced by Yao (1999), and used in an extreme value context by Daouia et al. (2011, 2013) and Escobar-Bach et al. (2018):

$$
c_{j}:=\arg \min _{c \in \mathcal{C}_{g}} \sum_{i=1}^{n} \sum_{k=1}^{n}\left[\mathbb{1}_{\left\{Y_{i}^{(j)} \leqslant Y_{k}^{(j)}\right\}}-\widetilde{F}_{n,-i, j}\left(Y_{k}^{(j)} \mid X_{i}\right)\right]^{2}, j=1,2,
$$

where $\mathcal{C}_{g}$ is a grid of values of $c$ and $\widetilde{F}_{n,-i, j}(y \mid x):=\frac{\sum_{k=1, k \neq i}^{n} K_{c}\left(x-X_{k}\right) 1_{\left\{Y_{k}^{(j)} \leqslant y\right\}}}{\sum_{k=1, k \neq i}^{n} K_{c}\left(x-X_{k}\right)}$. We take $\mathcal{C}_{g}=R_{X} \times\{0.05,0.10, \ldots, 0.30\}$, where $R_{X}$ is the range of the covariate $X$. The bandwidth parameter $h_{n}$ is determined from the condition

$$
n h_{n} \sqrt{\frac{|\log c|^{q}}{n c}} \longrightarrow 0
$$

by taking $h_{n}=R_{X} \sqrt{c /\left(n|\log c|^{\kappa}\right)}$, where $\kappa>q$ and $c:=\min \left(c_{1}, c_{2}\right)$. Next to $h_{n}$ and $c$, our estimation procedure also requires the selection of a threshold parameter $u_{n}$. As usual in extreme value statistics, this parameter will be set at the $(k+1)$-th largest of the $\check{Z}$ for which the $X$ coordinate is in $B\left(x, h_{n}\right)$.

As mentioned before, we only estimate $\eta(x)$ and $\delta_{Z}\left(u_{n} \mid x\right)$ with the MDPD method, while the parameter $\rho$ is fixed at some value. Here we set $\rho=-1$, which is a mis-specification.

For each of the above distributions we simulate $N=500$ samples of size $n=1000$. The results of the simulation experiment are reported in Figures 1 till 6 . In Figure 1 we show the mean of $\breve{\eta}_{n}(x)$ as a function of $k$ for $\alpha=0$ (solid line), $\alpha=0.5$ (dashed line) and $\alpha=1$ (dotted line) for the BB6 copula. The true value of $\eta$ is represented by the horizontal reference line. The columns of the figure represent three different values of $x$, while the rows correspond with the contamination percentages, $0 \%, 5 \%$ and $10 \%$ from top to bottom. Figure 2 displays the empirical mean squared error (MSE) as a function of $k$, but has otherwise a layout that is similar to Figure 1. Concerning the selection of $h_{n}$ and $c$, we note the following. In a first step we compute the optimal $h_{n}$ and $c$ for each dataset using the above mentioned cross-validation 
criterion. This implies that the range of $k$ varies from one dataset to the other, so means and MSE's would be based on a different number of observations when plotted as a function of $k$. In order to avoid this we take the median of the $h_{n}$ and $c$ values obtained in the 500 simulations and use this for all estimations. Figures 3 and 4, and Figures 5 and 6, show the corresponding results for the Farlie Gumbel Morgenstern and BB9 copula, respectively. From the simulation we can draw the following conclusions:

- In absence of contamination, the estimators show generally a quite stable pattern for a wide range of $k$, close to the true value of $\eta$, despite the mis-specification of the parameter $\rho$. In terms of MSE we see that, the estimator with $\alpha=0$, which corresponds to maximum likelihood, performs best, followed by $\alpha=0.5$ and $\alpha=1$. This can be explained as follows: in terms of bias the estimators with different values of $\alpha$ perform similarly, while for the variance we have that $\alpha=0$, corresponding to maximum likelihood, performs best. It is well-known that the efficiency of the MDPDE decreases with increasing $\alpha$, see, e.g., Basu et al. (1998).

- When there is contamination, then the non-robust estimator $(\alpha=0)$ is clearly affected, with a sample mean that can be far from the true value, while the robust estimators generally stay closer to the true value. The estimator with $\alpha=1$, which offers the highest robustness, performs best in terms of bias. In terms of minimal MSE, using $\alpha=0.5$ gives the best result. The advantage of $\alpha=1$ in terms of bias is offset by its increased variance compared to $\alpha=0.5$.

- The performance of the estimators deteriorates under increasing contamination percentages.

- For the BB6 distribution, the effect of the contamination is strongest for the smaller $x$ values. This could be expected, as the dependence in the data is weakest at the smaller $x$. The dependence increases with $x$, and therefore at $x=4$ the effect of contamination on the diagonal is least.

- The Farlie Gumbel Morgenstern distribution has $\eta=0.5$, corresponding to near independence. For this distribution, contamination on the diagonal is clearly very severe.

- For the BB9 distribution, which does not satisfy our model assumptions, we still have very good estimation results, which also illustrates the robustness of our methodology with respect to violation of the model assumption. Also here we see that the effect of the contamination is biggest at the $x$ positions where the dependence in the data is weakest.

- Overall, using $\alpha=0.5$ and 1 leads to estimators that are robust with respect to outliers. In terms of minimal MSE the estimator with $\alpha=0.5$ performs typically best, and is therefore the recommended value. This is in line with the findings of Dutang et al. (2014) in the context without covariates. 

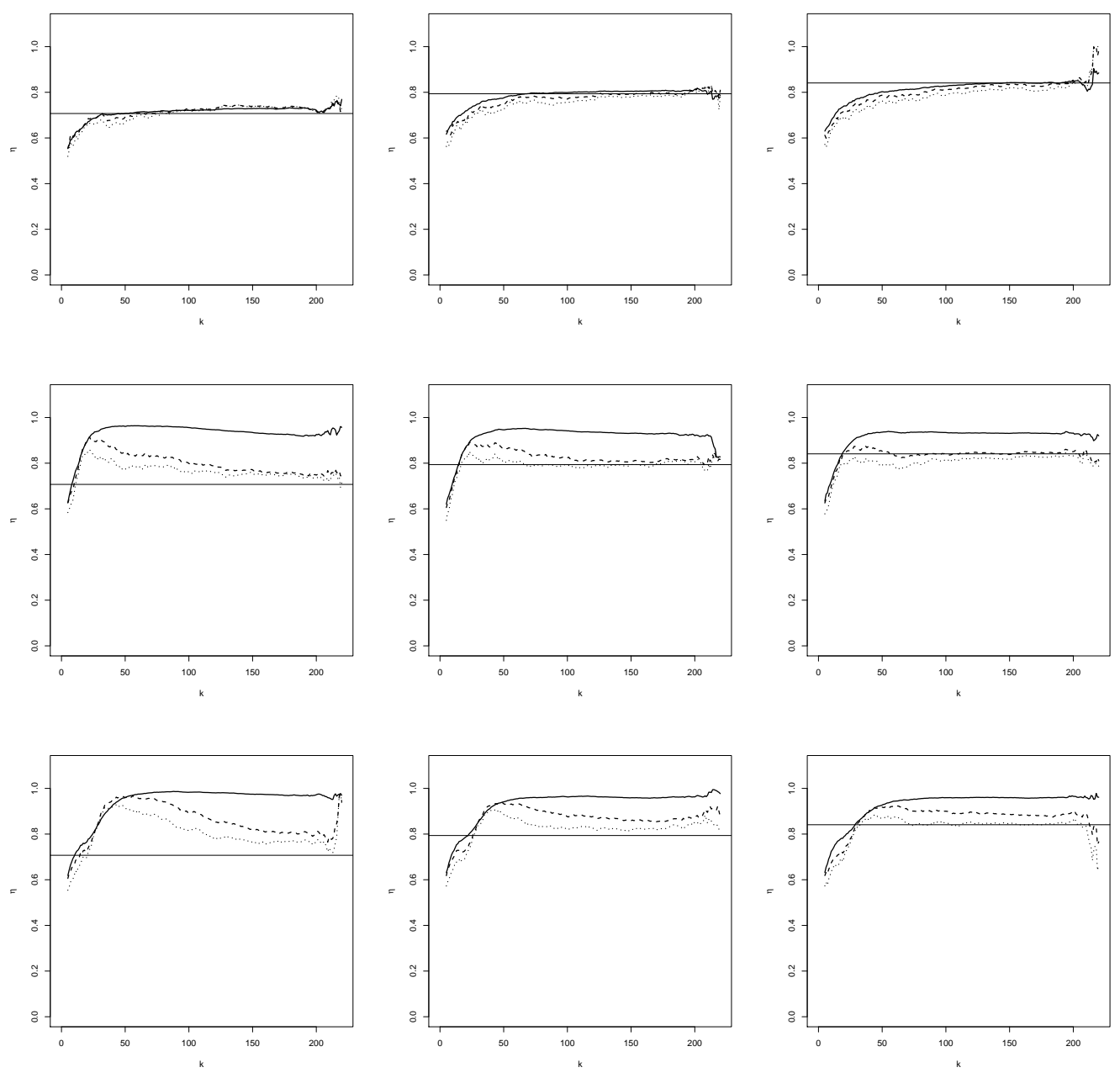

Figure 1: BB6 simulation with (shifted) diagonal contamination. Mean of $\breve{\eta}_{n}(x)$ with $\alpha=0$ (solid line), $\alpha=0.5$ (dashed line) and $\alpha=1$ (dotted line), as a function of $k$ at $x=2$ (left), $x=3$ (middle) and $x=4$ (right). From top to bottom: $0 \%, 5 \%$ and $10 \%$ of contamination.

\section{A real data analysis}

In this section, the proposed methodology is applied to a dataset of air pollution measurements. In environmental science, one needs to consider simultaneous high levels of several pollutants, possibly combined with high temperatures, as these may pose a major threat to human health. Estimation of the extreme dependence is thus of crucial importance in this context. We consider the data collected by the United States Environmental Protection Agency (EPA), publicly available at https://aqsdr1.epa.gov/aqsweb/aqstmp/airdata/download_files.html. The dataset under consideration contains monthly maxima on, among others, temperature, and ground-level ozone, 

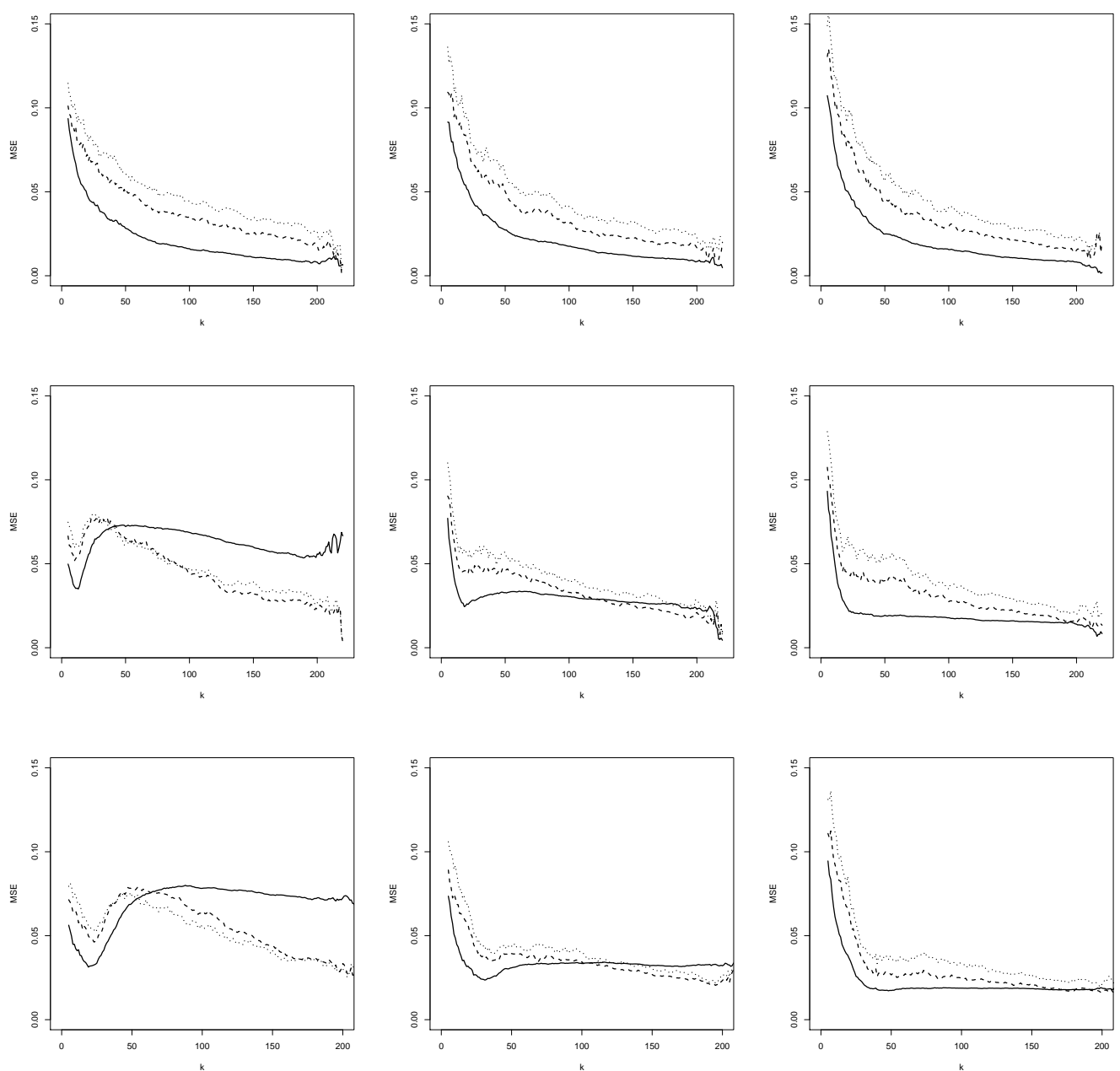

Figure 2: BB6 simulation with (shifted) diagonal contamination. MSE of $\breve{\eta}_{n}(x)$ with $\alpha=0$ (solid line), $\alpha=0.5$ (dashed line) and $\alpha=1$ (dotted line), as a function of $k$ at $x=2$ (left), $x=3$ (middle) and $x=4$ (right). From top to bottom: $0 \%, 5 \%$ and $10 \%$ of contamination.

carbon monoxide and particulate matter concentrations, for the time period 1999 to 2013. These data are collected at stations spread over the U.S. We will estimate the extreme dependence between ground-level ozone and particulate matter concentrations, conditional on the covariates time and location, where the latter is expressed by latitude and longitude. The method is implemented with the same cross-validation criteria as in the simulations, though for convenience we rescaled each covariate to the interval $[0,1]$. As for the kernel function, we used the bi-quadratic kernel, generalised to the case $p=3$, as follows

$$
K_{h_{n}}(x)=\frac{1}{h_{n}^{3}} K\left(\frac{\|x\|}{h_{n}}\right),
$$



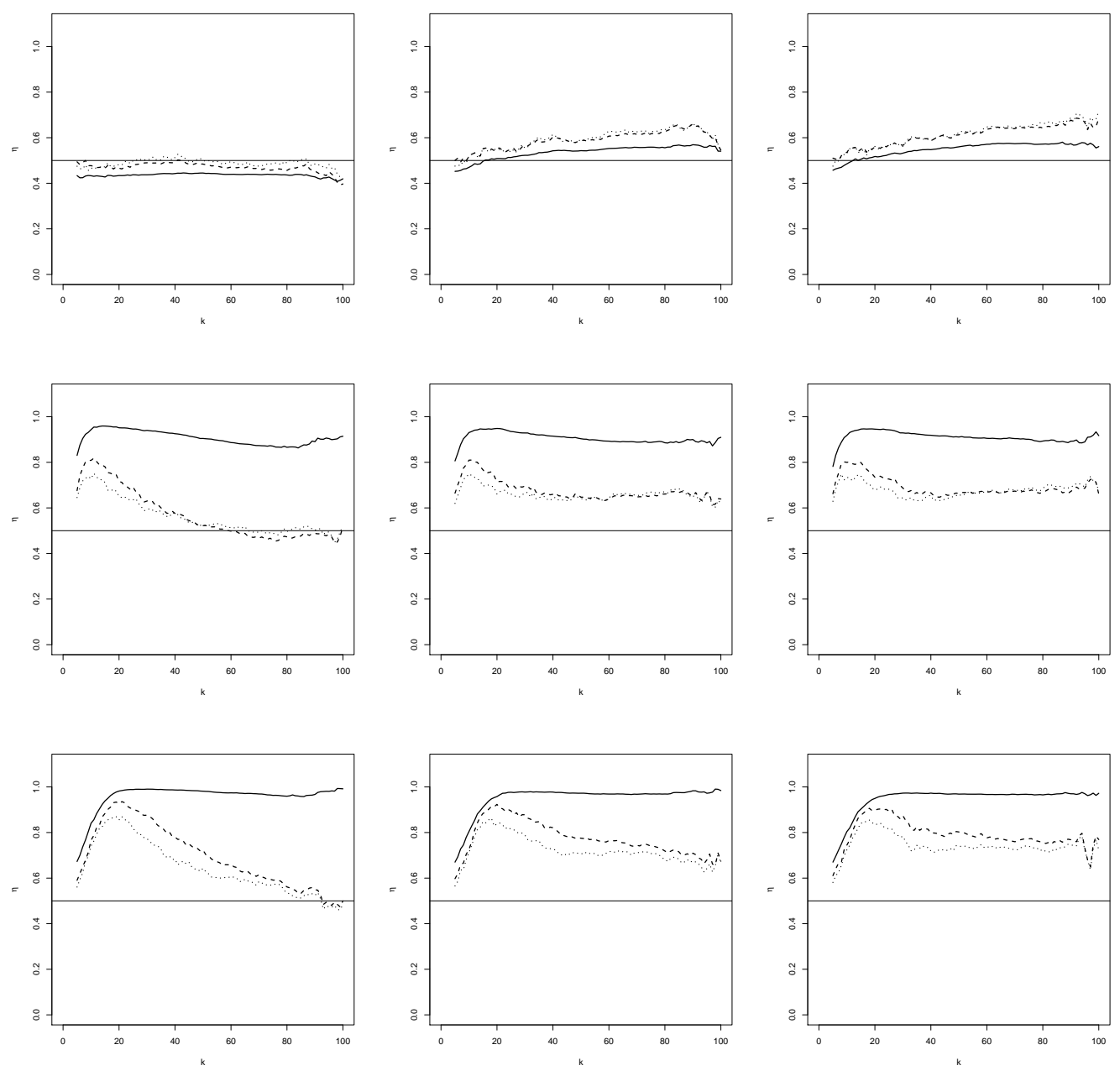

Figure 3: FGM simulation with (shifted) diagonal contamination. Mean of $\breve{\eta}_{n}(x)$ with $\alpha=0$ (solid line), $\alpha=0.5$ (dashed line) and $\alpha=1$ (dotted line), as a function of $k$ at $x=-0.5$ (left), $x=0.5$ (middle) and $x=0.8$ (right). From top to bottom: $0 \%, 5 \%$ and $10 \%$ of contamination.

where $x \in \mathbb{R}^{3}$, and $\|\cdot\|$ denotes the Euclidean norm. In Figure 7, we show the estimate of $\eta(x)$ with $\alpha=0$ (solid line), $\alpha=0.5$ (dashed line) and $\alpha=1$ (dotted line) for the city of Los Angeles at different points in time. The reported estimate is median $\left(\breve{\eta}_{n}(x) ; k=n^{*} / 2, \ldots, n^{*}-1\right)$, where $n^{*}$ denotes the number of observations in $B\left(x, h_{n}\right)$. Overall, the extreme dependence between ground-level ozone and particulate matter concentrations shows a seasonal pattern, where the dependence is stronger in summer than winter. For some months the estimate with $\alpha=0$ differs noticeably from those obtained with $\alpha=0.5$ and $\alpha=1$, which indicates the presence of observations that are disturbing for the estimation of the dependence structure. In Figure 8, we show the estimate $\breve{\eta}_{n}(x)$ with $\alpha=0$ (solid line), $\alpha=0.5$ (dashed line) and $\alpha=1$ (dotted line) 

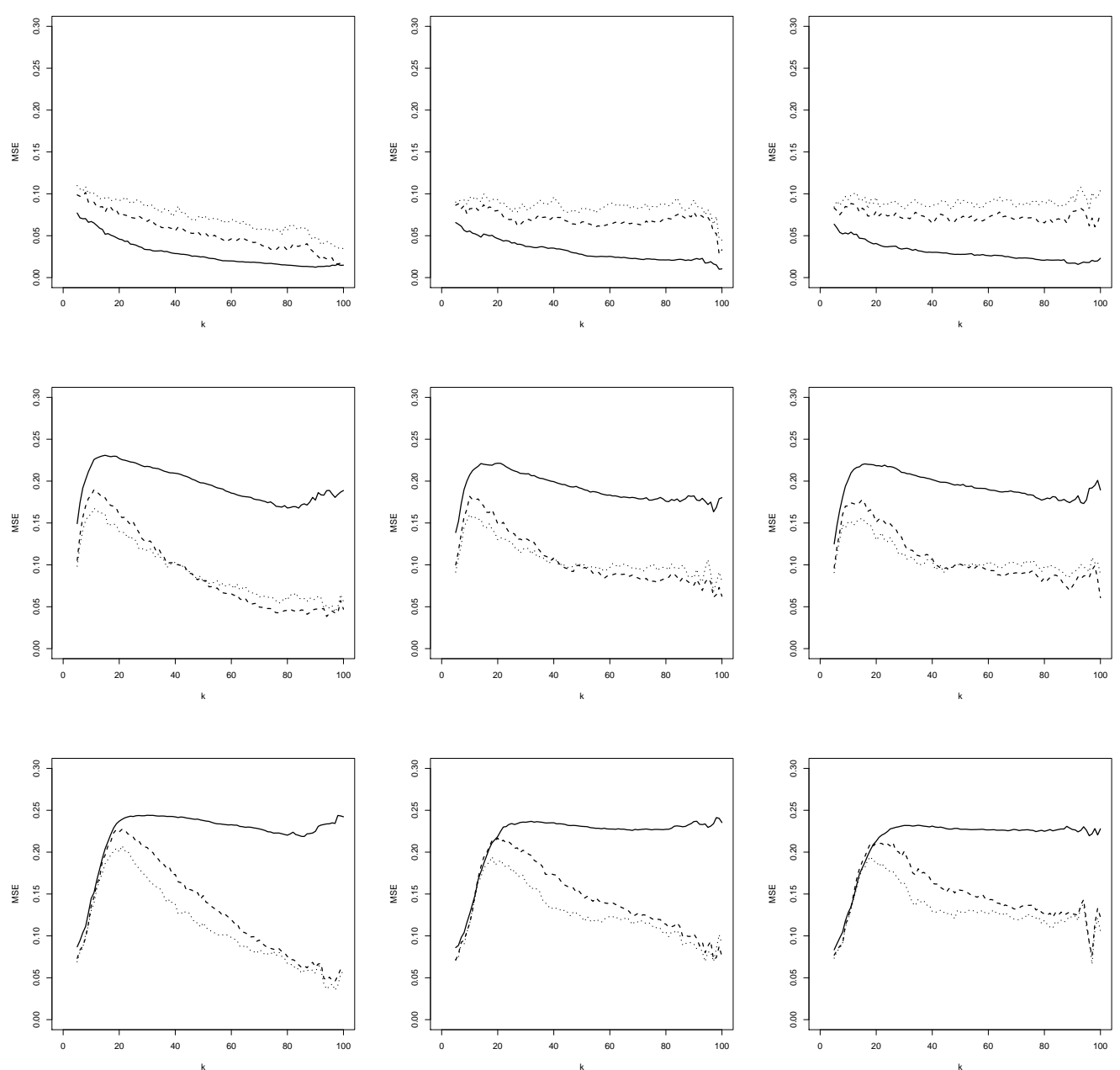

Figure 4: FGM simulation with (shifted) diagonal contamination. MSE of $\breve{\eta}_{n}(x)$ with $\alpha=0$ (solid line), $\alpha=0.5$ (dashed line) and $\alpha=1$ (dotted line), as a function of $k$ at $x=-0.5$ (left), $x=0.5$ (middle) and $x=0.8$ (right). From top to bottom: $0 \%, 5 \%$ and $10 \%$ of contamination.

for months 59 and 100 as a function of $k$. For month 59, the robust estimates show a stable pattern around $\eta(x)=1$ for the second half of the $k$ range, while the non-robust estimate shows nearly no stability as a function of $k$. On the contrary, for month 100, the robust estimates are below the non-robust estimate. Again the robust estimates show a stable horizontal pattern for the second half of the $k$ range, which is not present for the non-robust estimate. 

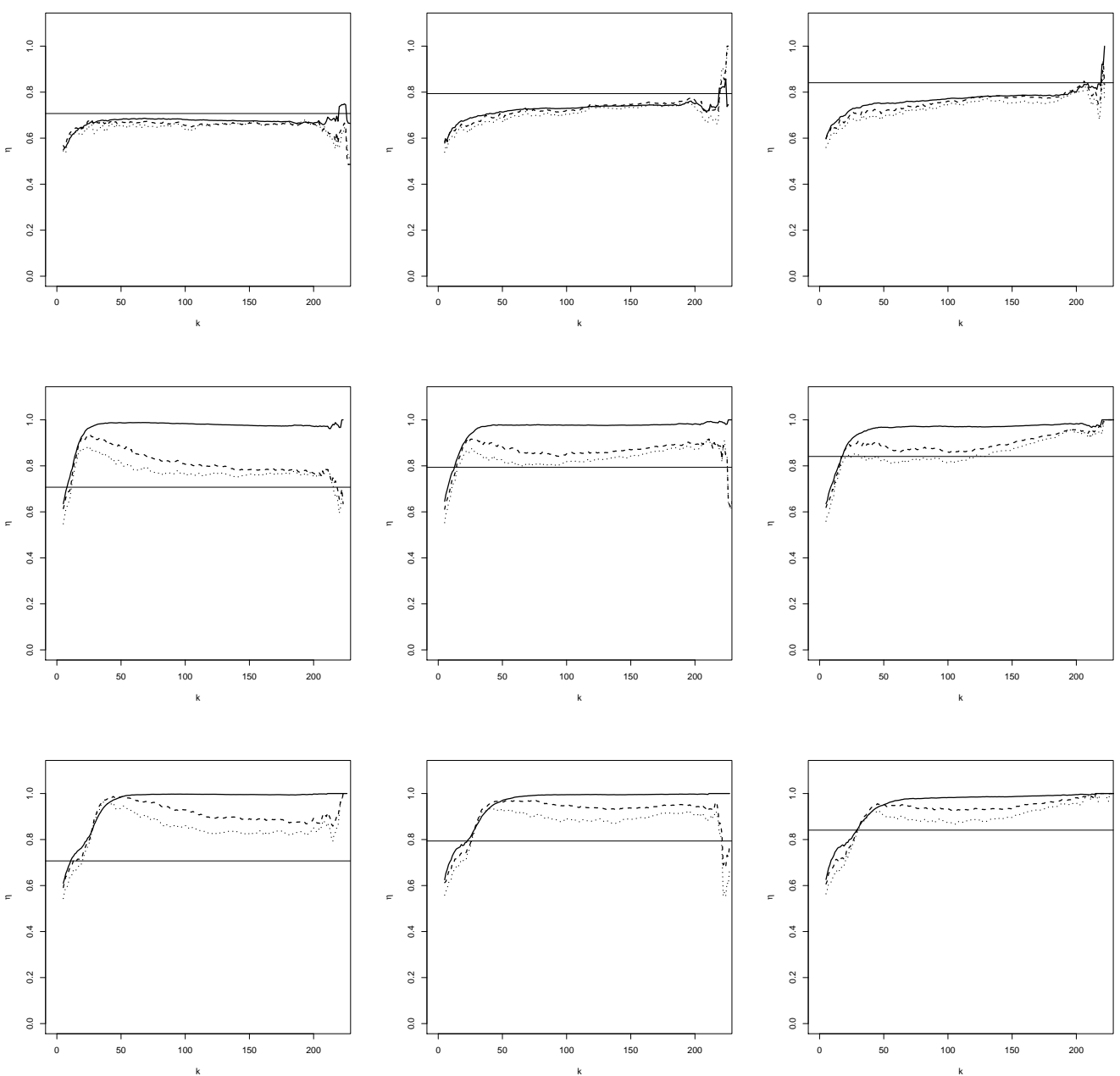

Figure 5: BB9 simulation with (shifted) diagonal contamination. Mean of $\breve{\eta}_{n}(x)$ with $\alpha=0$ (solid line), $\alpha=0.5$ (dashed line) and $\alpha=1$ (dotted line), as a function of $k$ at $x=2$ (left), $x=3$ (middle) and $x=4$ (right). From top to bottom: $0 \%, 5 \%$ and $10 \%$ of contamination.

\section{Appendix}

\subsection{Proof of Theorem 2.1}

The first step consists to show that, under our assumptions,

$$
\begin{aligned}
& \mathbb{E}\left[T_{n}(K, s, j \mid x)\right]=f_{X}(x) \bar{F}_{Z}\left(u_{n} \mid x\right) \eta_{0}^{j}(x) j ! \\
& \times\left\{\frac{1}{\left(1-s \eta_{0}(x)\right)^{j+1}}-\frac{\delta_{Z}\left(u_{n} \mid x\right)}{\eta_{0}(x)}\left[\frac{1}{\left(1-s \eta_{0}(x)\right)^{j+1}}-\frac{1-\rho_{0}(x)}{\left(1-\rho_{0}(x)-s \eta_{0}(x)\right)^{j+1}}\right]+o\left(\delta_{Z}\left(u_{n} \mid x\right)\right)\right. \\
& \left.\quad+O\left(h_{n}^{\delta_{f_{X}} \wedge \delta_{C}}\right)+O\left(h_{n}^{\delta_{\eta}} \ln u_{n}\right)\right\},
\end{aligned}
$$



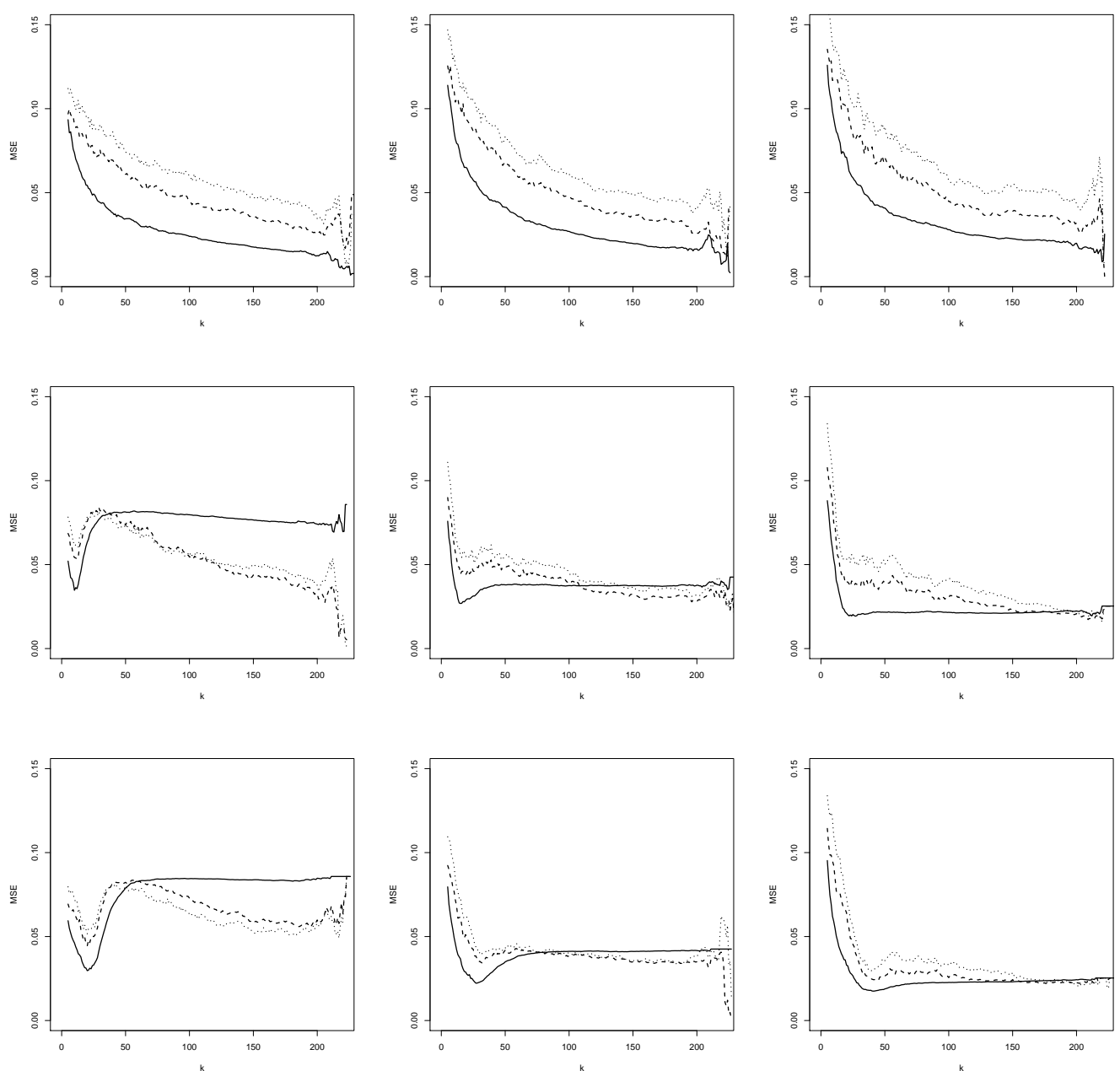

Figure 6: BB9 simulation with (shifted) diagonal contamination. MSE of $\breve{\eta}_{n}(x)$ with $\alpha=0$ (solid line), $\alpha=0.5$ (dashed line) and $\alpha=1$ (dotted line), as a function of $k$ at $x=2$ (left), $x=3$ (middle) and $x=4$ (right). From top to bottom: $0 \%, 5 \%$ and $10 \%$ of contamination.

where the $o\left(\delta_{Z}\left(u_{n} \mid x\right)\right)$ and $O($.$) terms are uniform in s \in[S, 0]$. 


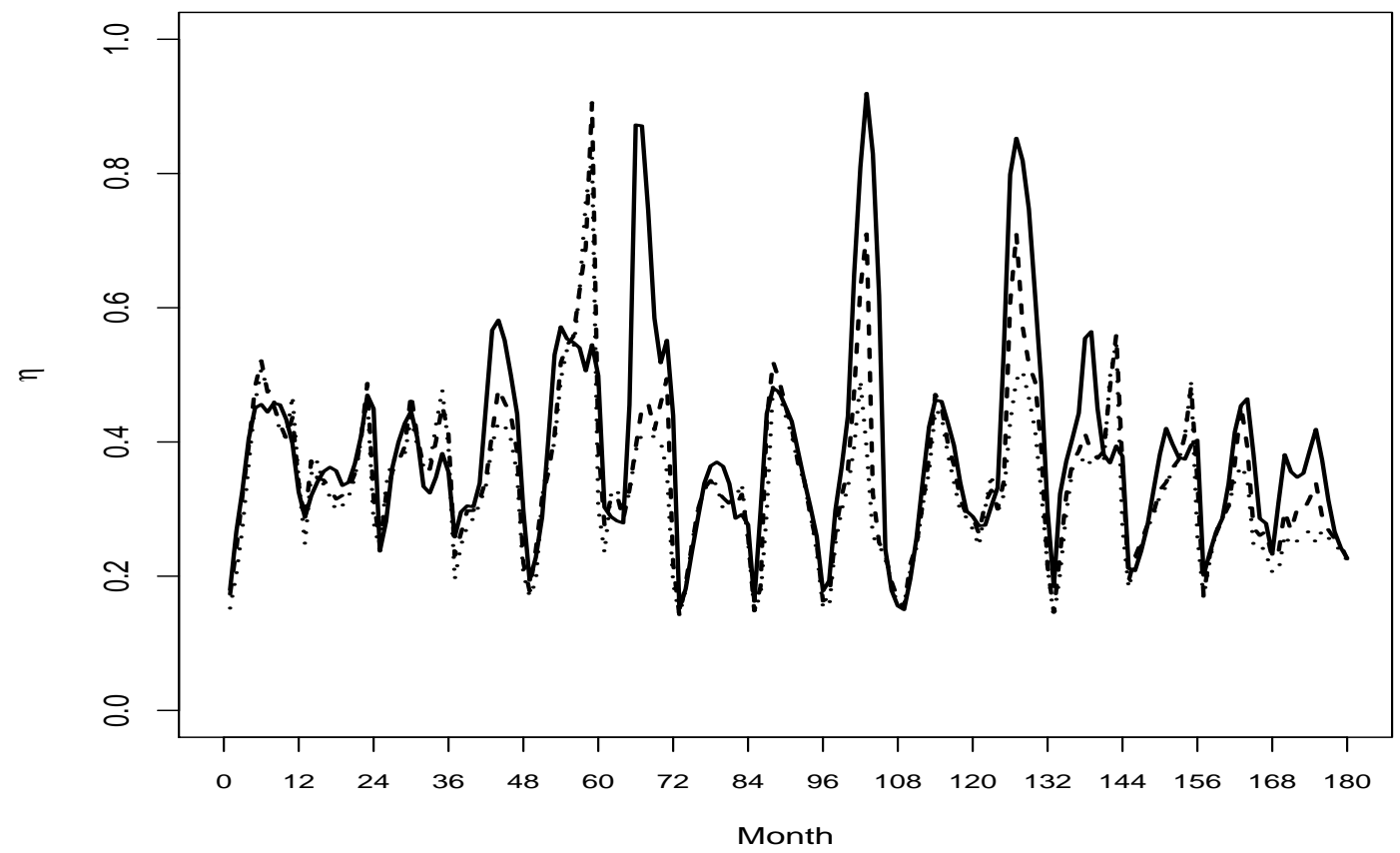

Figure 7: Air pollution data. Time plot of $\breve{\eta}_{n}(x)$ with $\alpha=0$ (solid line), $\alpha=0.5$ (dashed line) and $\alpha=1$ (dotted line) for the city of Los Angeles.

To obtain this result for the case $j>0$, use the following decomposition

$$
\begin{aligned}
\mathbb{E}\left[T_{n}(K, s, j \mid x)\right]= & f_{X}(x) \int_{1}^{\infty} p(z) \bar{F}_{Z}\left(u_{n} z \mid x\right) d z \\
& +\int_{S_{K}} K(v) \int_{1}^{\infty} p(z) \bar{F}_{Z}\left(u_{n} z \mid x\right) d z\left[f_{X}\left(x-h_{n} v\right)-f_{X}(x)\right] d v \\
& +f_{X}(x) \int_{S_{K}} K(v) \int_{1}^{\infty} p(z)\left[\bar{F}_{Z}\left(u_{n} z \mid x-h_{n} v\right)-\bar{F}_{Z}\left(u_{n} z \mid x\right)\right] d z d v \\
& +\int_{S_{K}} K(v) \int_{1}^{\infty} p(z)\left[\bar{F}_{Z}\left(u_{n} z \mid x-h_{n} v\right)-\bar{F}_{Z}\left(u_{n} z \mid x\right)\right] d z\left[f_{X}\left(x-h_{n} v\right)-f_{X}(x)\right] d v,
\end{aligned}
$$

where $p(z):=s z^{s-1}(\ln z)^{j}+j z^{s-1}(\ln z)^{j-1}$. Each term can be treated using our Hölder-type conditions, which imply in particular that, for $n$ large enough, $z \geqslant u_{n}$, and some constants $M_{1}, M_{2}, M_{3}$

$\left|\frac{\bar{F}_{Z}\left(z \mid x-h_{n} v\right)}{\bar{F}_{Z}(z \mid x)}-1\right| \leqslant M_{1}\left(h_{n}^{\delta_{C}}+z^{M_{2} h_{n}^{\delta_{\eta}}} h_{n}^{\delta_{\eta}} \ln z+\left|\delta_{Z}(z \mid x)\right| h_{n}^{\delta_{A}}+\left|\delta_{Z}(z \mid x)\right| z^{M_{3} h_{n}^{\delta_{\varepsilon}}} h_{n}^{\delta_{\varepsilon}} \ln z\right)$

combined with a slight modification of Proposition 2.3 in Beirlant et al. (2009) which ensures 

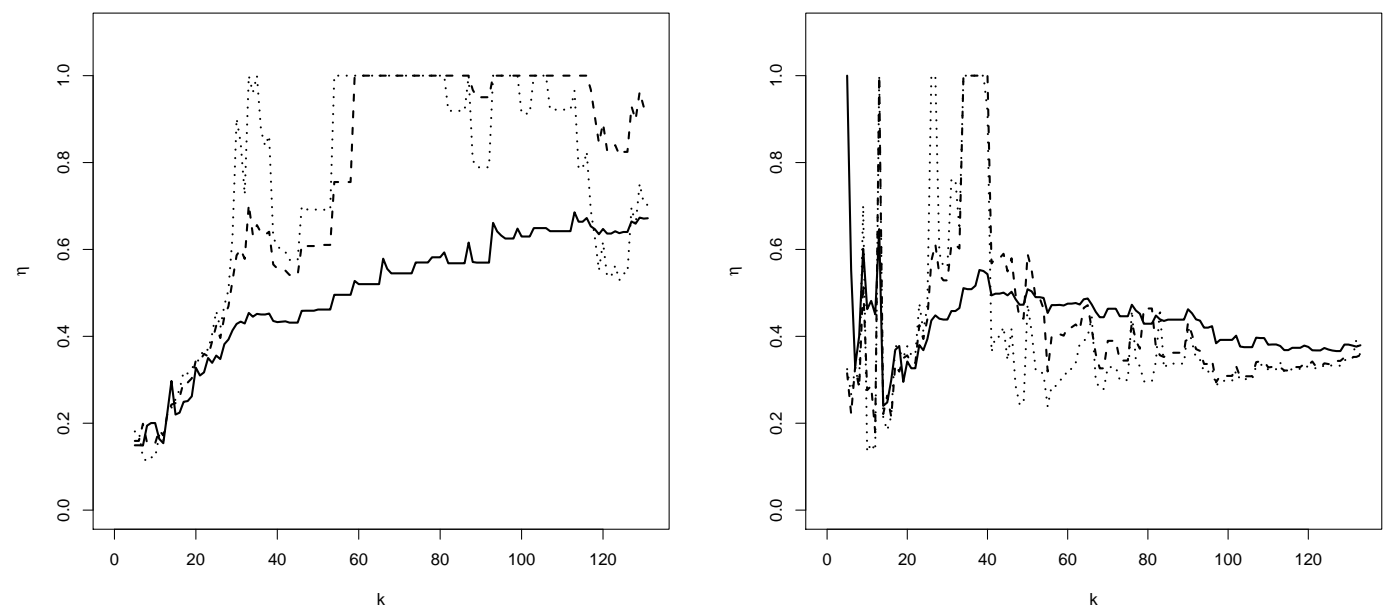

Figure 8: Air pollution data. $\check{\eta}_{n}(x)$ with $\alpha=0$ (solid line), $\alpha=0.5$ (dashed line) and $\alpha=1$ (dotted line) as a function of $k$ for months 59 (left) and 100 (right).

that

$$
\sup _{z \geqslant 1} z^{\frac{1}{\eta(x)}}\left|\frac{\bar{F}_{Z}\left(u_{n} z \mid x\right)}{\bar{F}_{Z}\left(u_{n} \mid x\right)}-\bar{G}\left(z ; \eta(x), \delta_{Z}\left(u_{n} \mid x\right), \rho(x)\right)\right|=o\left(\left|\delta_{Z}\left(u_{n} \mid x\right)\right|\right) \text { as } u_{n} \rightarrow \infty .
$$

In case $j=0$ we obtain

$$
\begin{aligned}
\mathbb{E}\left[T_{n}(K, s, 0 \mid x)\right]= & \int_{S_{K}} K(v) \int_{1}^{\infty} p(z) \bar{F}_{Z}\left(u_{n} z \mid x-h_{n} v\right) d z f_{X}\left(x-h_{n} v\right) d v \\
& +\int_{S_{K}} K(v) \bar{F}_{Z}\left(u_{n} \mid x-h_{n} v\right) f_{X}\left(x-h_{n} v\right) d v,
\end{aligned}
$$

where $p(z)=s z^{s-1}$. Both terms can be analysed with decompositions similar to the ones used for the case $j>0$.

Then we can follow the lines of proofs of Theorem 1 and Corollary 1 in Dierckx et al. (2014) in order to achieve the proof of Theorem 2.1.

\subsection{Proof of Theorem 3.1}

First remark that 


$$
\begin{aligned}
& \sqrt{\frac{n h_{n}^{p}}{\bar{F}_{Z}\left(u_{n} \mid x\right) f_{X}(x)}}\left[\breve{T}_{n}(K, s, j \mid x)-T_{n}(K, s, j \mid x)\right] \\
& =\quad \sqrt{\frac{n h_{n}^{p}}{\bar{F}_{Z}\left(u_{n} \mid x\right) f_{X}(x)}}\left[\breve{T}_{n}(K, s, j \mid x)-T_{n}(K, s, j \mid x)-\mathbb{E}\left(\breve{T}_{n}(K, s, j \mid x)-T_{n}(K, s, j \mid x)\right)\right] \\
& \quad+\sqrt{\frac{n h_{n}^{p}}{\bar{F}_{Z}\left(u_{n} \mid x\right) f_{X}(x)}} \mathbb{E}\left(\breve{T}_{n}(K, s, j \mid x)-T_{n}(K, s, j \mid x)\right) \\
& =: \quad R_{n, 1}+R_{n, 2} .
\end{aligned}
$$

We will study the two terms $R_{n, 1}$ and $R_{n, 2}$ separately. First, we start with the term $R_{n, 1}$. Define for any $s<0$ with $j \in J:=\{0,1,2,3\}$ or $(s, j)=(0,0)$

$$
g_{\xi, n}^{(s, j)}\left(y_{1}, y_{2}, v\right):=\sqrt{\frac{h_{n}^{p}}{\bar{F}_{Z}\left(u_{n} \mid x\right) f_{X}(x)}} K_{h_{n}}(x-v) q_{\xi, n}^{(s, j)}\left(y_{1}, y_{2}, v\right)
$$

with

$$
\begin{aligned}
q_{\xi, n}^{(s, j)}\left(y_{1}, y_{2}, v\right) & :=\left(\frac{Z_{\xi}\left(y_{1}, y_{2}, v\right)}{u_{n}}\right)^{s}\left(\ln \frac{Z_{\xi}\left(y_{1}, y_{2}, v\right)}{u_{n}}\right)^{j} \mathbb{1}_{\left\{Z_{\xi}\left(y_{1}, y_{2}, v\right)>u_{n}\right\}}, \\
Z_{\xi}\left(y_{1}, y_{2}, v\right) & :=\min \left(\frac{1}{\left|1-\xi_{1}\left(y_{1}, y_{2}, v\right)\right|}, \frac{1}{\left|1-\xi_{2}\left(y_{1}, y_{2}, v\right)\right|}\right)
\end{aligned}
$$

and measurable $\xi \in H:=\left\{\xi=\left(\xi_{1}, \xi_{2}\right) ; \xi: \mathbb{R} \times \mathbb{R} \times S_{X} \rightarrow \mathbb{R}^{2}\right\}$.

For convenience, denote $\xi_{n}=\left(F_{n, 1}, F_{n, 2}\right)$ and $\xi_{0}=\left(F_{1}, F_{2}\right)$. According to Lemma $3.1, r_{n}^{-1}\left|\xi_{n}-\xi_{0}\right|$ converges in probability towards the null function $H_{0}=\{0\}$ in $H$, endowed with the norm $\|\xi\|_{H}:=\left\|\xi_{1}\right\|_{\infty}+\left\|\xi_{2}\right\|_{\infty}$ for any $\xi \in H$. Consider now the class

$$
\mathcal{E}_{n}^{(s, j)}(b):=\left\{g_{\xi_{0}+r_{n} \xi, n}^{(s, j)}-g_{\xi_{0}, n}^{(s, j)}: \xi \in H,\|\xi\|_{H} \leqslant b\right\},
$$

with envelope function $G_{n}^{(s, j)}(b)$. Our aim is to apply Theorem 2.3 in van der Vaart and Wellner (2017). To reach this goal, we need to introduce some notations. Let $P$ denote the law of the vector $\left(Y^{(1)}, Y^{(2)}, X\right)$ and define the expectation of any real-valued measurable function $f$ under $P$ by $P f=\int f d P$.

We have now to show the two following results:

Assertion 1: For any $s<0$ with $j \in J$ or $(s, j)=(0,0)$, we have

$$
\sqrt{n} P G_{n}^{(s, j)}\left(b_{n}\right) \longrightarrow 0 \text { for all } b_{n} \rightarrow 0,
$$

and

Assertion 2: For any $s<0$ with $j \in J$ or $(s, j)=(0,0)$, we have

$$
P\left(\left(G_{n}^{(s, j)}(b)\right)^{2}\right) \longrightarrow 0 .
$$




\subsubsection{Proof of Assertion 1}

We start to consider the case where $s<0$ with $j \in J$. As a first step we derive an envelope function for $\mathcal{E}_{n}^{(s, j)}\left(b_{n}\right)$. We have

$$
\begin{aligned}
& \left|q_{\xi_{0}+r_{n} \xi, n}^{(s, j)}-q_{\xi_{0}, n}^{(s, j)}\right| \\
& =\mid \int_{1}^{\infty} p(a) \mathbb{1}_{\left\{u_{n}<u_{n} a<Z_{\xi_{0}+r_{n} \xi}\right\}} \mathbb{1}_{\left\{Z_{\xi_{0}+r_{n} \xi}>u_{n}\right\}} d a-\int_{1}^{\infty} p(a) \mathbb{1}_{\left\{u_{n}<u_{n} a<Z_{\xi_{0}}\right\}} \mathbb{1}_{\left\{Z_{\xi_{0}}>u_{n}\right\}} d a \\
& +\mathbb{1}_{\{j=0\}} \mathbb{1}_{\left\{Z_{\xi_{0}+r_{n} \xi}>u_{n}\right\}}-\mathbb{1}_{\{j=0\}} \mathbb{1}_{\left\{Z_{\xi_{0}}>u_{n}\right\}} \mid \\
& \leqslant \int_{1}^{\infty}|p(a)|\left|\mathbb{1}_{\left\{u_{n}<u_{n} a<Z_{\xi_{0}+r_{n} \xi}\right\}} \mathbb{1}_{\left\{Z_{\xi_{0}+r_{n} \xi}>u_{n}\right\}}-\mathbb{1}_{\left\{u_{n}<u_{n} a<Z_{\xi_{0}}\right\}} \mathbb{1}_{\left\{Z_{\xi_{0}}>u_{n}\right\}}\right| d a \\
& +\mathbb{1}_{\{j=0\}}\left|\mathbb{1}_{\left\{Z_{\xi_{0}+r_{n} \xi}>u_{n}\right\}}-\mathbb{1}_{\left\{Z_{\xi_{0}}>u_{n}\right\}}\right| \\
& \leqslant \int_{1}^{\infty}|p(a)|\left|\mathbb{1}_{\left\{u_{n}<u_{n} a<Z_{\xi_{0}}\right\}}-\mathbb{1}_{\left\{u_{n}<u_{n} a<Z_{\xi_{0}+r_{n} \xi}\right\}}\right| \mathbb{1}_{\left\{Z_{\xi_{0}}>u_{n}\right\}} d a \\
& +\int_{1}^{\infty}|p(a)| \mathbb{1}_{\left\{u_{n}<u_{n} a<Z_{\left.\xi_{0}+r_{n} \xi\right\}}\right\}}\left|\mathbb{1}_{\left\{Z_{\xi_{0}+r_{n} \xi}>u_{n}\right\}}-\mathbb{1}_{\left\{Z_{\xi_{0}}>u_{n}\right\}}\right| d a \\
& +\mathbb{1}_{\{j=0\}}\left|\mathbb{1}_{\left\{Z_{\xi_{0}+r_{n} \xi}>u_{n}\right\}}-\mathbb{1}_{\left\{Z_{\xi_{0}}>u_{n}\right\}}\right| \\
& \leqslant \int_{1}^{\infty}|p(a)| \mathbb{1}_{\left\{\min \left(Z_{\xi_{0}}, Z_{\xi_{0}+r_{n} \xi}\right) \leqslant u_{n} a \leqslant \max \left(Z_{\xi_{0}}, Z_{\xi_{0}+r_{n} \xi}\right)\right\}} \mathbb{1}_{\left\{Z_{\xi_{0}}>u_{n}\right\}} d a \\
& +\int_{1}^{\infty}|p(a)| \mathbb{1}_{\left\{u_{n}<u_{n} a<Z_{\xi_{0}+r_{n} \xi}\right\}} \mathbb{1}_{\left\{\min \left(Z_{\xi_{0}}, Z_{\xi_{0}+r_{n} \xi}\right) \leqslant u_{n} \leqslant \max \left(Z_{\xi_{0}}, Z_{\xi_{0}+r_{n} \xi}\right)\right\}} d a \\
& +1_{\{j=0\}} 1_{\left\{\min \left(Z_{\xi_{0}}, Z_{\xi_{0}+r_{n} \xi}\right) \leqslant u_{n} \leqslant \max \left(Z_{\xi_{0}}, Z_{\xi_{0}+r_{n} \xi}\right)\right\}} \text {. }
\end{aligned}
$$

Remark now that 
$\left\{u_{n} a \in\left[\min \left(Z_{\xi_{0}}, Z_{\xi_{0}+r_{n} \xi}\right) ; \max \left(Z_{\xi_{0}}, Z_{\xi_{0}+r_{n} \xi}\right)\right]\right\}$

$$
\begin{aligned}
= & \left\{u _ { n } a \in \left[\min \left(\min \left(\frac{1}{\left|1-F_{1}-r_{n} \xi_{1}\right|}, \frac{1}{\left|1-F_{2}-r_{n} \xi_{2}\right|}\right), \min \left(\frac{1}{1-F_{1}}, \frac{1}{1-F_{2}}\right)\right) ;\right.\right. \\
= & \left.\left.\max \left(\min \left(\frac{1}{\left|1-F_{1}-r_{n} \xi_{1}\right|}, \frac{1}{\left|1-F_{2}-r_{n} \xi_{2}\right|}\right), \min \left(\frac{1}{1-F_{1}}, \frac{1}{1-F_{2}}\right)\right)\right]\right\} \\
\subset & \left\{\frac { 1 } { u _ { n } a } \in \left[\min \left(\max \left(\left|1-F_{1}-r_{n} \xi_{1}\right|,\left|1-F_{2}-r_{n} \xi_{2}\right|\right), \max \left(1-F_{1}, 1-F_{2}\right)\right) ;\right.\right. \\
& \cup\left\{\frac{1}{u_{n} a} \in\left[\max \left(\max \left(\left|1-F_{1}-r_{n} \xi_{1}\right|,\left|1-r_{n} \xi_{1}\right|, 1-F_{n}\right), \max \left(\left|1-F_{n}\right|\right), \max \left(1-F_{1}-F_{1}-r_{n} \xi_{1}\left|, 1-F_{2} \xi_{2}\right|, 1-F_{1}\right)\right]\right\}\right. \\
\subset & \left.\left\{\frac{1}{u_{n} a} \in\left[1-F_{2}\right), \max \left(\left|1-F_{2}-r_{n} \xi_{2}\right|, 1-F_{2}\right)\right]\right\} \\
=: & A_{n, 1}(a) \cup A_{n, 2}(a) .
\end{aligned}
$$

Also,

$$
\begin{aligned}
\mathbb{1}_{\left\{Z_{\xi_{0}+r_{n} \xi}>u_{n} a\right\}} & =\mathbb{1}_{\left\{\left|1-F_{1}-r_{n} \xi_{1}\right|<\frac{1}{u_{n} a},\left|1-F_{2}-r_{n} \xi_{2}\right|<\frac{1}{u_{n} a}\right\}} \\
& \leqslant 1_{\left\{-\frac{1}{u_{n} a}-r_{n} b_{n}<1-F_{1}<\frac{1}{u_{n} a}+r_{n} b_{n},-\frac{1}{u_{n} a}-r_{n} b_{n}<1-F_{2}<\frac{1}{u_{n} a}+r_{n} b_{n}\right\}} \\
& =\mathbb{1}_{\left\{1-F_{1}<\frac{1}{u_{n} a}+r_{n} b_{n}, 1-F_{2}<\frac{1}{u_{n} a}+r_{n} b_{n}\right\}},
\end{aligned}
$$

and, taking into account that

$$
\mathbb{1}_{\left\{Z_{\xi_{0}}>u_{n}\right\}}=\mathbb{1}_{\left\{1-F_{1}<\frac{1}{u_{n}}, 1-F_{2}<\frac{1}{u_{n}}\right\}}
$$

we obtain

$$
\begin{aligned}
\mathbb{1}_{\left\{Z_{\xi_{0}+r_{n} \xi}>u_{n} a\right\}} & \leqslant \mathbb{1}_{\left\{1-F_{1}<\frac{1}{u_{n}}\left(\frac{1}{a}+r_{n} u_{n} b_{n}\right), 1-F_{2}<\frac{1}{u_{n}}\left(\frac{1}{a}+r_{n} u_{n} b_{n}\right)\right\}} \\
& =\mathbb{1}_{\left\{Z_{\xi_{0}}>\frac{u_{n}}{\frac{1}{a}+r_{n} u_{n} b_{n}}\right\}} .
\end{aligned}
$$

Thus, combining (13), (14) and (15), we obtain the following envelope for $\mathcal{E}_{n}^{(s, j)}\left(b_{n}\right)$ :

$$
\begin{aligned}
G_{n}^{(s, j)}\left(b_{n}\right):= & \sqrt{\frac{h_{n}^{p}}{\bar{F}_{Z}\left(u_{n} \mid x\right) f_{X}(x)}} K_{h_{n}}(x-.)\left[\int_{1}^{\infty}|p(a)| \mathbb{1}_{\left\{A_{n, 1}(a) \cup A_{n, 2}(a)\right\}} \mathbb{1}_{\left\{Z_{\xi_{0}}>u_{n}\right\}} d a\right. \\
& \left.+\int_{1}^{\infty}|p(a)| \mathbb{1}_{\left\{Z_{\xi_{0}}>\frac{u_{n}}{\frac{1}{a}+r_{n} u_{n} b_{n}}\right.}\right\} \\
& +\mathbb{1}_{\{j=0\}} \mathbb{1}_{\left\{A_{n, 1}(1) \cup A_{n, 2}(1)\right\}},
\end{aligned}
$$


with

$$
\begin{aligned}
& \sqrt{n} P G_{n}^{(s, j)}\left(b_{n}\right)=\sqrt{\frac{n h_{n}^{p}}{\bar{F}_{Z}\left(u_{n} \mid x\right) f_{X}(x)}} \times \\
& \left\{\mathbb{E}\left[K_{h_{n}}(x-X) \int_{1}^{\infty}|p(a)| \mathbb{E}\left(\mathbb{1}_{\left\{A_{n, 1}(a) \cup A_{n, 2}(a)\right\}} \mathbb{1}_{\left\{Z_{\xi_{0}}>u_{n}\right\}} \mid X\right) d a\right]\right. \\
& +\mathbb{E}\left[K_{h_{n}}(x-X) \int_{1}^{\infty}|p(a)| \mathbb{E}\left(\mathbb{1}_{\left\{Z_{\xi_{0}>\frac{u_{n}}{\frac{1}{a}+r_{n} u_{n} b_{n}}}\right\}} \mathbb{1}_{\left\{A_{n, 1}(1) \cup A_{n, 2}(1)\right\}} \mid X\right) d a\right] \\
& \left.+\mathbb{1}_{\{j=0\}} \mathbb{E}\left[K_{h_{n}}(x-X) \mathbb{E}\left(\mathbb{1}_{\left\{A_{n, 1}(1) \cup A_{n, 2}(1)\right\}} \mid X\right)\right]\right\} \\
& =\sqrt{\frac{n h_{n}^{p}}{\bar{F}_{Z}\left(u_{n} \mid x\right) f_{X}(x)}} \times \\
& \left\{\int_{S_{K}} K(v) \int_{1}^{\infty}|p(a)| \mathbb{E}\left(\mathbb{1}_{\left\{A_{n, 1}(a) \cup A_{n, 2}(a)\right\}} \mathbb{1}_{\left\{Z_{\xi_{0}}>u_{n}\right\}} \mid X=x-h_{n} v\right) d a f_{X}\left(x-h_{n} v\right) d v\right. \\
& +\int_{S_{K}} K(v) \int_{1}^{\infty}|p(a)| \mathbb{E}\left(\mathbb{1}_{\left\{Z_{\xi_{0}}>\frac{u_{n}}{\frac{1}{a}+r_{n} u_{n} b_{n}}\right\}} \mathbb{1}_{\left\{A_{n, 1}(1) \cup A_{n, 2}(1)\right\}} \mid X=x-h_{n} v\right) d a f_{X}\left(x-h_{n} v\right) d v \\
& \left.+\mathbb{1}_{\{j=0\}} \int_{S_{K}} K(v) \mathbb{E}\left(\mathbb{1}_{\left\{A_{n, 1}(1) \cup A_{n, 2}(1)\right\}} \mid X=x-h_{n} v\right) f_{X}\left(x-h_{n} v\right) d v\right\} \\
& =: \sqrt{\frac{n h_{n}^{p}}{\bar{F}_{Z}\left(u_{n} \mid x\right) f_{X}(x)}}\left\{T_{1}+T_{2}+T_{3}\right\} .
\end{aligned}
$$

Consider $T_{1}$. By the Cauchy-Schwarz inequality

$T_{1} \leqslant \int_{S_{K}} K(v) \int_{1}^{\infty}|p(a)| \sqrt{\mathbb{P}\left(A_{n, 1}(a) \cup A_{n, 2}(a) \mid X=x-h_{n} v\right) \bar{F}_{Z}\left(u_{n} \mid x-h_{n} v\right)} d a f_{X}\left(x-h_{n} v\right) d v$.

The sub-additivity of probability measures and some straightforward calculations give then

$$
\begin{aligned}
\mathbb{P}\left(A_{n, 1}(a) \cup A_{n, 2}(a) \mid X=x-h_{n} v\right) & \leqslant \mathbb{P}\left(A_{n, 1}(a) \mid X=x-h_{n} v\right)+\mathbb{P}\left(A_{n, 2}(a) \mid X=x-h_{n} v\right) \\
& =\int_{0}^{1} 1_{\left\{\frac{1}{u_{n} a} \in\left[z-r_{n} b_{n}, z+r_{n} b_{n}\right]\right\}} d z+\int_{0}^{1} \mathbb{1}_{\left\{\frac{1}{u_{n} a} \in\left[z-r_{n} b_{n}, z+r_{n} b_{n}\right]\right\}} d z \\
& \leqslant 2 r_{n} b_{n}+2 r_{n} b_{n}=4 r_{n} b_{n} .
\end{aligned}
$$

Thus

$$
T_{1} \leqslant 2 \sqrt{r_{n} b_{n} \bar{F}_{Z}\left(u_{n} \mid x\right)} \int_{1}^{\infty}|p(a)| d a \int_{S_{K}} K(v) \sqrt{\frac{\bar{F}_{Z}\left(u_{n} \mid x-h_{n} v\right)}{\bar{F}_{Z}\left(u_{n} \mid x\right)}} f_{X}\left(x-h_{n} v\right) d v
$$

and hence, by (12) and the Hölder continuity of $f_{X}$, we have $T_{1}=O\left(\sqrt{r_{n} \bar{F}_{Z}\left(u_{n} \mid x\right)}\right)$. 
As for $T_{2}$ use again the Cauchy-Schwarz inequality and (17) to obtain

$$
\begin{aligned}
T_{2} & \leqslant 2 \sqrt{r_{n} b_{n}} \int_{1}^{\infty}|p(a)| d a \int_{S_{K}} K(v) \sqrt{\bar{F}_{Z}\left(\frac{u_{n}}{1+r_{n} u_{n} b_{n}} \mid x-h_{n} v\right)} f_{X}\left(x-h_{n} v\right) d v \\
& =2 \sqrt{r_{n} b_{n} \bar{F}_{Z}\left(\frac{u_{n}}{1+r_{n} u_{n} b_{n}} \mid x\right)} \int_{1}^{\infty}|p(a)| d a \int_{S_{K}} K(v) \sqrt{\frac{\bar{F}_{Z}\left(\frac{u_{n}}{1+r_{n} u_{n} b_{n}} \mid x-h_{n} v\right)}{\bar{F}_{Z}\left(\frac{u_{n}}{1+r_{n} u_{n} b_{n}} \mid x\right)}} f_{X}\left(x-h_{n} v\right) d v .
\end{aligned}
$$

Note that under our assumptions, $r_{n} u_{n} \rightarrow 0$, as $n \rightarrow \infty$. Thus using (12) and the fact that

$$
\frac{\bar{F}_{Z}\left(\frac{u_{n}}{1+r_{n} u_{n} b_{n}} \mid x\right)}{\bar{F}_{Z}\left(u_{n} \mid x\right)} \rightarrow 1,
$$

we have that $T_{2}=O\left(\sqrt{r_{n} \bar{F}_{Z}\left(u_{n} \mid x\right)}\right)$.

By similar arguments, we get $T_{3}=O\left(r_{n}\right)=o\left(\sqrt{r_{n} \bar{F}_{Z}\left(u_{n} \mid x\right)}\right)$ under our assumptions (10) and (11).

Combining the above

$$
\sqrt{n} P G_{n}^{(s, j)}\left(b_{n}\right)=O\left(\sqrt{n h_{n}^{p} r_{n}}\right) .
$$

Now, we move to the case $(s, j)=(0,0)$ and use a similar proof. In that case, using (17), we have

$$
\begin{aligned}
\sqrt{n} P G_{n}^{(0,0)}\left(b_{n}\right) & \\
\quad= & \sqrt{\frac{n h_{n}^{p}}{\bar{F}_{Z}\left(u_{n} \mid x\right) f_{X}(x)}} \int_{S_{K}} K(v) \mathbb{P}\left(A_{n, 1}(1) \cup A_{n, 2}(1) \mid X=x-h_{n} v\right) f_{X}\left(x-h_{n} v\right) d v \\
& \leqslant 4 r_{n} b_{n} \sqrt{\frac{n h_{n}^{p}}{\bar{F}_{Z}\left(u_{n} \mid x\right) f_{X}(x)}} \int_{S_{K}} K(v) f_{X}\left(x-h_{n} v\right) d v \\
& =O\left(\sqrt{\frac{n h_{n}^{p}}{\bar{F}_{Z}\left(u_{n} \mid x\right)}} r_{n} b_{n}\right) \\
& =o\left(\sqrt{n h_{n}^{p} r_{n}}\right)
\end{aligned}
$$

which achieves the proof of Assertion 1 in case $(s, j)=(0,0)$. 


\subsubsection{Proof of Assertion 2}

Again, we start to look at the case $s<0$ and $j \in J$. From (16) and straightforward bounds, we deduce that

$$
\begin{aligned}
\left(G_{n}^{(s, j)}(b)\right)^{2} \leqslant & \bar{F}_{Z}\left(u_{n} \mid x\right) f_{X}(x) \\
& \times\left\{\left(\int_{h_{n}}^{2} \mid x-\cdot\right)\right. \\
& \left.+3\left(\int_{1}^{\infty}|p(a)| d a\right) \int_{1}^{\infty}|p(a)| \mathbb{1}_{\left\{Z_{\xi_{0}}>\frac{u_{n}}{\frac{1}{a}+r_{n} u_{n} b}\right\}}\right\} \mathbb{1}_{\left\{A_{n, 1}(1) \cup A_{n, 2}(1)\right\}} d a \\
& +\left(1+4 \int_{1}^{\infty}|p(a)| \mathbb{1}_{\left\{A_{n, 1}(a) \cup A_{n, 2}(a)\right\}} \mid \mathbb{1}_{\left\{Z_{\left.\xi_{0}>u_{n}\right\}}\right.} d a\right.
\end{aligned}
$$

Since $\int_{1}^{\infty}|p(a)| d a<\infty$, using again the Cauchy-Schwarz inequality combined with (17), we deduce that

$$
\begin{aligned}
& P\left(\left(G_{n}^{(s, j)}(b)\right)^{2}\right) \\
& \leqslant \frac{C \sqrt{r_{n}}}{\bar{F}_{Z}\left(u_{n} \mid x\right) f_{X}(x)} \int_{S_{K}} K^{2}(v) \int_{1}^{\infty}|p(a)| \sqrt{\bar{F}_{Z}\left(\frac{u_{n}}{\frac{1}{a}+r_{n} u_{n} b} \mid x-h_{n} v\right)} d a f_{X}\left(x-h_{n} v\right) d v \\
& \quad+\frac{C \sqrt{r_{n}}}{\bar{F}_{Z}\left(u_{n} \mid x\right) f_{X}(x)} \int_{S_{K}} K^{2}(v) \int_{1}^{\infty}|p(a)| \sqrt{\bar{F}_{Z}\left(u_{n} \mid x-h_{n} v\right)} d a f_{X}\left(x-h_{n} v\right) d v \\
& \quad+\frac{C r_{n}}{\bar{F}_{Z}\left(u_{n} \mid x\right) f_{X}(x)} \int_{S_{K}} K^{2}(v) f_{X}\left(x-h_{n} v\right) d v
\end{aligned}
$$

where $C$ is a constant which can change from one line to each other.

Finally, combining (12) with (18), we deduce that

$$
P\left(\left(G_{n}^{(s, j)}(b)\right)^{2}\right)=O\left(\sqrt{\frac{r_{n}}{\bar{F}_{Z}\left(u_{n} \mid x\right)}}\right) .
$$

The case $(s, j)=(0,0)$ can be dealt with similarly and leads to

$$
P\left(\left(G_{n}^{(0,0)}(b)\right)^{2}\right)=O\left(\frac{r_{n}}{\bar{F}_{Z}\left(u_{n} \mid x\right)}\right) .
$$

This achieves the proof of Assertion 2.

Combining Assertions 1 and 2 with Theorem 2.3 in van der Vaart and Wellner (2017) yields that $R_{n, 1}=o_{\mathbb{P}}(1)$. 
Now, it remains to study the term $R_{n, 2}$. To this aim, note that, for $n$ large,

$$
\begin{aligned}
\left|R_{n, 2}\right| & \leqslant \sqrt{\frac{n h_{n}^{p}}{\bar{F}_{Z}\left(u_{n} \mid x\right) f_{X}(x)}} \mathbb{E}\left|\breve{T}_{n}(K, s, j \mid x)-T_{n}(K, s, j \mid x)\right| \\
& \leqslant \sqrt{n} \mathbb{E}\left|g_{\xi_{n}, n}^{(s, j)}\left(Y^{(1)}, Y^{(2)}, X\right)-g_{\xi_{0}, n}^{(s, j)}\left(Y^{(1)}, Y^{(2)}, X\right)\right| \\
& \leqslant \sqrt{n} P G_{n}^{(s, j)}(b),
\end{aligned}
$$

since $\xi_{n} \in \xi_{0}+r_{n} \mathcal{B}(0, b)$ where $\mathcal{B}(0, b):=\left\{\xi:\|\xi\|_{H} \leqslant b\right\}$ (where we use the Skorohod representation). According to the proof of Assertion 1, since $b_{n} \rightarrow 0$ can be replaced by any fixed value $b$ without changing the conclusion, we have $R_{n, 2}=o(1)$.

Combining the results for $R_{n, 1}$ and $R_{n, 2}$ achieves the proof of Theorem 3.1.

\section{References}

Barbe, P., Fougères, A.-L. and Genest, C. (2006). On the tail behavior of sums of dependent risks. Astin Bull., 36, 361-373.

Basu, A., Harris, I.R., Hjort, N.L. and Jones, M.C. (1998). Robust and efficient estimation by minimizing a density power divergence. Biometrika, 85, 549-559.

Beirlant, J., Dierckx, G., Goegebeur, Y. and Matthys, G. (1999). Tail index estimation and an exponential regression model. Extremes, 2, 177-200.

Beirlant, J., Dierckx, G. and Guillou, A. (2011). Bias-reduced estimators for bivariate tail modelling. Insurance Math. Econom., 49, 18-26.

Beirlant, J., Goegebeur, Y., Segers, J. and Teugels, J. (2004). Statistics of Extremes - Theory and Applications. Wiley.

Beirlant, J., Joossens, E. and Segers, J. (2009). Second-order refined peaks-over-threshold modelling for heavy-tailed distributions. J. Statist. Plann. Inference, 139, 2800-2815.

Daouia, A., Gardes, L. and Girard, S. (2013). On kernel smoothing for extremal quantile regression. Bernoulli, 19, 2557-2589.

Daouia, A., Gardes, L., Girard, S. and Lekina, A. (2011). Kernel estimators of extreme level curves. TEST, 20, 311-333.

Dierckx, G., Goegebeur, Y. and Guillou, A. (2014). Local robust and asymptotically unbiased estimation of conditional Pareto type-tails. TEST, 23, 330-355.

Draisma, G., Drees, H., Ferreira, A. and de Haan, L., (2004). Bivariate tail estimation: dependence in asymptotic independence. Bernoulli, 10, 251-280.

Dutang, C., Goegebeur, Y. and Guillou, A. (2014). Robust and unbiased estimation of the coefficient of tail dependence. Insurance Math. Econom., 57, 46-57. 
Escobar-Bach, M., Goegebeur, Y. and Guillou, A. (2018). Local robust estimation of the Pickands dependence function. Ann. Statist., 46, 2806-2843.

Feuerverger, A. and Hall, P. (1999). Estimating a tail exponent by modelling departure from a Pareto distribution. Ann. Statist., 27, 760-781.

Giné, E. and Guillou, A. (2002). Rates of strong uniform consistency for multivariate kernel density estimators. Ann. Inst. Henri Poincaré Probab. Stat., 38, 907-921.

Giné, E., Koltchinskii, V. and Zinn, J. (2004). Weighted uniform consistency of kernel density estimators. Ann. Probab., 32, 2570-2605.

Goegebeur, Y. and Guillou, A. (2013). Asymptotically unbiased estimation of the coefficient of tail dependence. Scand. J. Stat., 40, 174-189.

Gomes, M.I. and Martins, M.J. (2004). Bias reduction and explicit estimation of the extreme value index. J. Statist. Plann. Inference, 124, 361-378.

de Haan, L. and Ferreira, A. (2006). Extreme Value Theory: An Introduction. Springer.

Joe, H. (1997). Multivariate models and dependence concepts. Chapman and Hall, London.

Ledford, A.W. and Tawn, J.A. (1997). Modelling dependence within joint tail regions. J. Roy. Statist. Soc. Ser. B, 59, 475-499.

Peng, L. (1999). Estimation of the coefficient of tail dependence in bivariate extremes. Statist. Probab. Lett., 43, 399-409.

van der Vaart, A. W. and Wellner, J. A. (2007). Empirical processes indexed by estimated functions, Asymptotics: Particles, Processes and Inverse Problems, IMS Lecture Notes Monogr. Ser., 55, 234-252.

Yao, Q. (1999). Conditional predictive regions for stochastic processes. Technical report, University of Kent at Canterbury. 